\title{
MARKOV PROCESSES AND RANDOM FIELDS ${ }^{1}$
}

\section{Introduction.}

BY E. B. DYNKIN

1.1. Suppose that a particle moves in a space $E$ under the influence of random factors. Its position $x_{t}$ at time $t$ is a random variable, that is a measurable function on a space $\Omega$ where a probability measure $P$ is given. The family $X=\left\{x_{t}\right\}$ is called a stochastic process in the state space $E$. It is important to evaluate the future behaviour of the particle using, in the best possible way, the information available at the present time. A stochastic process $X$ is Markovian if, for a given value of $x_{t_{0}}$, the prognosis of the future does not depend on the evolution before $t_{0}$. A more symmetric form of the same property is: the families $x_{t}, t>t_{0}$ and $x_{t}, t<t_{0}$ are conditionally independent given $x_{t_{0}}$. During the past decades Markov processes became a powerful tool in partial differential equations and potential theory with important applications to physics.

Recently a growing interest is attracted by a generalization of stochastic processes known as random fields. A random field $\Phi$ over a space $E$ is a family of random variables $\varphi_{x}, x \in E$. This is a mathematical model for systems with a large number of interacting random components which arise in physics, biology, sociology, theory of automata, etc.

A random field $\Phi$ over a space $E$ has the Markov property on a pair of subsets $B, C$ of $E$ if the values of $\Phi$ on $B$ and on $C$ are conditionally independent given the values on the intersection $B \cap C$.

Investigation of the Markov property of a random field is closely related to the following prediction problem: To evaluate the values of the field on a set $C$ by functionals of its values on a set $B$. A field has the Markov property on $B, C$ if and only if the best estimate of values on $C$ by values on $B$ is a functional of values on $B \cap C$.

More precisely, we consider the Hilbert space $L^{2}(\Omega, P)$. Elements of this space which are determined by the values of $\Phi$ on $B$ form a subspace $L(B)$. The best estimate of $Y \in L^{2}(\Omega, P)$ by an element of $L(B)$ is, geometrically, the orthogonal projection of $Y$ on $L(B)$; in probabilistic language, it is called the conditional mathematical expectation of $Y$ given $\Phi$ on $B$.

Suppose that random variables $\varphi_{x}$ are real-valued and let $H$ be the subspace of $L^{2}(\Omega, P)$ linearly generated by $\varphi_{x}, x \in E$. There exists an important class of fields, called Gaussian fields (see the definition at the

Address delivered at the 765th meeting of the American Mathematical Society (New York, April 1979) and at the meeting of London Mathematical Society (the Hardy Lecture, June 1979). The results of 87 are more recent; received by the editors April 15, 1980.

1980 Mathematics Subject Classification. Primary 06G60; Secondary 60G20, 60G55, 81C20.

Key words and phrases. Markov property of Gaussian random fields, prediction problem, symmetric Markov processes, families of noninteracting Markov processes, additive functionals, stopping times.

${ }^{1}$ Research supported by NSF Grant No. MCS 77-03543. 
beginning of §2), for which the conditioning preserves $H$. This facilitates greatly the investigation of Gaussian fields.

The Markov property of Gaussian random fields has been studied by many authors: McKean [18], Molchan [20], [21] and others. Nelson [22], [23] has shown that the Markov property of certain random fields on Euclidean spaces has important implications for quantum field theory (see also [28]).

In this paper we introduce a class of Gaussian random fields associated with families of symmetric Markov processes and we investigate the prediction problem for such fields using paths of the Markov processes.

In particular, the Nelson free Markov field is associated with the exponentially killed Brownian motion. The Brownian sheet, studied by Yeh [34], Orey and Pruitt [25], Cairoli and Walsh [3], Walsh [29], Wong and Zakai [33] and others, is associated with two (or several) Brownian motions on a positive half-line $(0,+\infty)$ killed at the first hitting time of the origin.

Prerequisites for reading the article are a general knowledge of measure theory and understanding the measure-theoretical meaning of such terms as a probability space, the mathematical expectation of a random variable, the joint probability distribution of several random variables (see e.g. [1] or [17]). Beyond this we define all the probabilistic terms which we use.

1.2. A standard way to define a Markov process is to give the probability $p_{t}(x, B)$ of the transition from a point $x$ to the set $B$ in time $t$. We consider the case when

$$
p_{t}(x, B)=\int_{B} p_{t}(x, y) m(d y)
$$

where $m$ is a measure on the state space $E$ (given on a $\sigma$-algebra $\mathscr{B}$ ). The function $p_{t}(x, y)$ (subject to conditions listed in $\$ 3$ ) is called the transition density. We say that a Markov process is symmetric if $p_{t}(x, y)=p_{t}(y, x)$ for all $t, x, y$.

To every $x \in E$ there corresponds a measure $P_{x}$ on the space $\Omega$-the probability law of the motion starting from the point $x$. If $\mu$ is a measure on $E$, then the integral of $P_{x}$ with respect to $\mu$ is the law $P_{\mu}$ corresponding to initial measure $\mu$.

An important example of a symmetric transition density in the $d$-dimensional Euclidean space $R^{d}$ (relative to the Lebesgue measure $m$ ) is the function

$$
p_{t}(x, y)=e^{-t \lambda}(2 \pi t)^{-d / 2} \exp \left(-(1 / 2 t)|y-x|^{2}\right)
$$

where $|y-x|$ is the Euclidean distance between $x$ and $y$ and $\lambda>0$ is a constant. The corresponding Markov process is called the exponentially killed Brownian motion ( $\lambda$ describes the killing rate).

For the Brownian motion on a positive half-line

$$
p_{t}(x, y)=(2 \pi t)^{-1 / 2}\left[\exp \left(-(1 / 2 t)|y-x|^{2}\right)-\exp \left(-(1 / 2 t)|y+x|^{2}\right)\right]
$$

The function

$$
g(x, y)=\int_{0}^{\infty} p_{t}(x, y) d t
$$


is called the Green function. For the density (1.2)

$$
g(x, y)=\min (x, y)
$$

and for the density (1.1) with $d=1$

$$
g(x, y)=(2 \lambda)^{-1 / 2} \exp [-\sqrt{2 \lambda}|x-y|] \text {. }
$$

If $\boldsymbol{g}$ is finite, then there exists a Gaussian field indexed by $x \in E$ such that $E \varphi_{x}=0$,

$$
E \varphi_{x} \varphi_{y}=g(x, y) .
$$

However for the most interesting densities like the Brownian density (1.1) with $d>1$, the Green function is infinite for $x=y$ and no Gaussian field satisfying (1.3) exists.

To overcome this difficulty, we introduce a field indexed by measures.

Put

$$
\langle\mu, \nu\rangle=\int_{E \times E} \mu(d x) g(x, y) \nu(d y)
$$

and let $\mathscr{T}$ stand for the set of all $\sigma$-finite measures $\mu$ on $(E, \mathscr{B})$ for which $\langle\mu, \mu\rangle\left\langle\infty\right.$. There exists a Gaussian field $\Phi=\left\{\varphi_{\mu}, \mu \in \mathscr{N}\right\}$ such that $E \varphi_{\mu}=0$,

$$
E \varphi_{\mu} \varphi_{\nu}=\langle\mu, \nu\rangle .
$$

We call $\Phi$ the Gaussian field associated with the Markov process $X^{2}{ }^{2}$

In the case of a finite Green function, we can define $\varphi_{\mu}$ by the formula

$$
\varphi_{\mu}=\int_{E} \varphi_{x} \mu(d x)
$$

It follows from (1.5) that

$$
\varphi_{\mu+\nu}=\varphi_{\mu}+\varphi_{\nu} \text { a.s., } \quad \varphi_{c \mu}=c \varphi_{\mu} \text { a.s. }
$$

for every positive constant $c$.

Traditionally, functions rather than measures are used to index random fields. In our notations, this means that $\varphi_{\mu}$ is considered only for $\mu$ absolutely continuous with respect to $m$. Since every $m \in \mathscr{R}$ can be approximated in metric (1.5) by absolutely continuous measures, there is no fundamental difference between the two approaches. But the theory based on measures is simpler in many respects.

Denote by $\mathscr{T}(B)$ the set of all $\mu$ of $\Re$ which do not charge the complement of $B$. The family $\Phi_{B}=\left\{\varphi_{\mu}, \mu \in \mathfrak{R}(B)\right\}$ describes values of the field $\Phi$ on the set $B .^{3}$

Under certain regularity conditions for the process $X$, the following two results will be proved in \$6:

THEOREM 1.2.1. The Gaussian field associated with a symmetric Markov

\footnotetext{
${ }^{2}$ There exists no relation between the probability spaces on which $\Phi$ and $X$ are defined.

${ }^{3} \mathrm{~A}$ similar definition is introduced in a recent paper of $\mathbf{S}$. Albeverio and $\mathbf{R}$. Hoegh-Krohn (Comm. Math. Phys. 68 (1979), 95-128). Traditionally, to define $\Phi_{B}$ a topology in $E$ is used, a direct definition is given only for open sets $B$, and closed sets are treated by passage to the limit.
} 
process $X$ has the Markov property on all sets $B, C$ such that:

1.2. A. It is impossible to reach $C$ from $B$ without crossing $B \cap C$.

THEOREM 1.2.2. The conditional mathematical expectation of $\varphi_{\mu}$ given $\Phi_{B}$ is $\varphi_{\mu_{B}}$ where $\mu_{B}$ is the probability distribution of $x_{\tau}$ at the first hitting time $\tau$ of $B$ assuming that the initial probability distribution is $\mu^{4}$

The Brownian motion has the property 1.2.A for every two closed sets covering $E$. The same is true for every process with continuous paths in a topological space. Note that the property 1.2.A is symmetric in $B$ and $C$ because of the symmetry of $p_{t}(x, y)$.

Theorem 1.2.2 solves the prediction problem for an arbitrary set $B \in \mathscr{B}$.

1.3. The Gaussian field $\Phi$ associated with a family $X$ of symmetric Markov processes $X^{1}, X^{2}, \ldots, X^{k}$ is defined on the product $(E, \mathscr{B}, m)$ of the state spaces $\left(E^{i}, \mathscr{B}^{i}, m^{i}\right), i=1,2, \ldots, k$. Put

$$
\begin{aligned}
& g(x, y)=g^{1}\left(x^{1}, y^{1}\right) \ldots g^{k}\left(x^{k}, y^{k}\right) \\
& \qquad \text { for } x=\left(x^{1}, \ldots, x^{k}\right), y=\left(y^{1}, \ldots, y^{k}\right)
\end{aligned}
$$

where $g^{i}\left(x^{i}, y^{i}\right)$ is the Green function of $X^{i}$. The field $\Phi$ is defined by (1.4) and (1.5). Now $\mathfrak{T}$ is a class of measures on the product space $(E, \mathscr{B})$.

To investigate $\Phi$, we consider a path $x_{t^{i}}^{i}\left(\omega^{i}\right), t^{i}>0, \omega^{i} \in \Omega^{i}$ of the process $X^{i}$ and we put

$$
x_{t}(\omega)=\left(x_{t^{1}}^{1}\left(\omega^{1}\right), \ldots, x_{t^{k}}^{k}\left(\omega^{k}\right)\right) \text { for } \omega=\left(\omega^{1}, \ldots, \omega^{k}\right), t=\left(t^{1}, \ldots, t^{k}\right) .
$$

The multidimensional time parameter $t$ takes values in the product $T=$ $[0, \infty] \times \cdots \times[0, \infty]$ of $k$ positive half-lines. Put $s<t$ if $s^{1}<t^{1}, \ldots, s^{k}<$ $t^{k}$. For $k=1$, this is the standard ordering of positive numbers. For $k>1$, the ordering of $T$ is only partial. Because of this, the first hitting time of a set $B \subset E$ by $X$ exists only for a limited class of sets $B$.

It does exist for rectangles $B=B^{1} \times \cdots \times B^{k}$ : if $\tau^{i}$ is the first hitting time of $B^{i}$ by $X^{i}$, then $\tau=\left(\tau^{1}, \ldots, \tau^{k}\right)$ satisfies the conditions: if $x_{t} \in B$ then $t>\tau$ and for every $u>\tau$, there is a $0<t<u$ such that $x_{t} \in B$. Theorem 1.2.2 is applicable to this case and gives the solution of the prediction problem for rectangles.

For other sets the solution is more complicated. Sometimes it has a form $\varphi_{\mu^{\prime}}-\varphi_{\mu^{\prime \prime}}, \mu^{\prime}, \mu^{\prime \prime} \in \mathfrak{N}$. We extend the index set for $\Phi$ to $\tilde{\mathfrak{K}}=\mathfrak{T}-\mathfrak{N}$ putting $\varphi_{\mu^{\prime}-\mu^{\prime \prime}}=\varphi_{\mu^{\prime}}-\varphi_{\mu^{\prime \prime}}$ which is possible since the equality $\mu^{\prime}-\mu^{\prime \prime}=\nu^{\prime}-$ $\nu^{\prime \prime}$ implies the equality $\varphi_{\mu^{\prime}}-\varphi_{\mu^{\prime \prime}}=\varphi_{\nu^{\prime}}-\varphi_{\nu^{\prime \prime}}$ by linearity of $\varphi_{\mu}$. Generally, the conditional mathematical expectation $E\left(\varphi_{\mu} \mid \Phi_{B}\right)$ is the limit in quadratic mean of $\varphi_{\mu_{n}}$ for a sequence $\mu_{n} \in \tilde{\mathscr{T}}$.

We continue the bilinear form (1.4) to $\tilde{\mathfrak{K}}$. For every $\mu \in \tilde{\mathfrak{K}},\langle\mu, \mu\rangle>0$ and we put $\|\mu\|=\sqrt{\langle\mu, \mu\rangle}$.

1.4. We call a set elementary if it can be represented as a finite union of rectangles. The prediction problem for elementary sets can be solved using suitable families of stopping times.

Put $s<t$ if $s^{i}<t^{i}$ for every $i$. A stopping time $\tau$ is a random element of $T$

${ }^{4}$ The "probability distribution" here could be any $\sigma$-finite measure. 
with the property: for every $t \in T$, the event $\{\tau<t\}$ depends only on $x_{s}$, $s<t$. If $\tau^{i}$ is a stopping time for $X^{i}, i=1, \ldots, k$, then $\tau=\left(\tau^{1}, \ldots, \tau^{k}\right)$ is a stopping time for $X$ (the converse is not true).

Let $Q$ be a finite or a countable set and let a stopping time $\tau_{q}$ and a random variable $Z_{q}$ be defined for every $q \in Q$. We say that $\left(\tau_{q}, Z_{q}\right)$ is a $B$-resolving system if:

1.4.A. $Z_{q}$ depends only on $x_{t}, t<\tau_{q}$.

1.4.B. For every $\omega$ and $t$, either $x_{t}(\omega) \notin B$ or

$$
\sum_{\tau_{q} \in \mathscr{I}_{i}} Z_{q}=1
$$

Here $\mathscr{Z}_{t}$ is the set of all $\tau_{q}$ such that $\tau_{q}<t$ and $x_{\tau_{q}} \in B$.

The following result is a generalization of Theorem 1.2.2.

THEOREM 1.4.1. Let $\left(\tau_{q}, Z_{q}\right)$ be a B-resolving system and let $\tilde{Z}_{q}=Z_{q} 1_{B}\left(x_{\tau}\right)$. Let $P_{\mu}$ stand for the measure on $\Omega$ corresponding to the initial distribution $\mu \in \mathfrak{N}$. To every $q \in Q$ there correspond a signed measure ${ }^{5}$

$$
\mu_{q}(C)=P_{\mu} \tilde{Z}_{q} 1_{C}\left(x_{\tau_{q}}\right)
$$

and a positive measure

$$
\hat{\mu}_{q}(C)=P_{\mu}\left|\tilde{Z}_{q}\right| 1_{C}\left(x_{\tau_{q}}\right)
$$

If

$$
\sum_{q \in Q}\left\|\mu_{q}\right\|<\infty
$$

then

$$
E\left(\varphi_{\mu} \mid \Phi_{B}\right)=\sum_{q \in Q} \varphi_{\mu_{\varphi}}
$$

(the series converges in quadratic mean).

In 87 we give a general method of constructing resolving systems for elementary sets. We also describe simpler resolving systems for interesting particular cases (like the complement of a rectangle).

1.5. The prediction problem for nonelementary sets is postponed to a later publication. Here we only mention that the following approach is possible. Geometrically, $E\left\{\varphi_{\mu} \mid \Phi_{B}\right\}$ is the orthogonal projection of $\varphi_{\mu}$ on the space $H(B)$ linearly generated by $\Phi_{B}$. Let $H^{+}(B)$ stand for the intersection of $H(C)$ over all elementary sets $C$ which contain $B$. If the orthogonal projections of $\varphi_{\mu}$ on all $H(C)$ are known, then the projection on $H^{+}(B)$ can be obtained by passage to the limit. Under certain conditions, the limit can be described using integration. However $H^{+}(B)$ is generally larger than $H(B)$ and the evaluation of the orthogonal projections on $H(B)$ and on $H^{+}(B)$ are closely related but different problems. Up to now, these problems were studied only for the Brownian sheet. The study was initiated by Walsh [29]; the most complete results were recently obtained by Wolpert [32].

\footnotetext{
${ }^{5}$ If $P$ is a measure and $Z$ is a measurable function, then $P Z$ means the integral of $Z$ with respect to $P$.
} 
The prediction problem is closely connected with the Dirichlet problem for certain differential equations. If $X$ is the $d$-dimensional Brownian motion and if $\tau_{B}$ is the first hitting time of a closed set $B$, then the function

$$
F(x)=P_{x} f\left(x_{\tau_{b}}\right)
$$

is a generalized solution of the Dirichlet problem for the Laplace equation $\Delta F=0$ in the domain $E \backslash B$ with the boundary function $f$ (see e.g. [7, Chapter 2]). For a diffusion process $X$ this is true with the Laplacian $\Delta$ replaced by the infinitesimal generator $D$ of $X$ (which is an elliptic differential operator).

Let now $\left(\tau_{q}, Z_{q}\right)$ be a $B$-resolving system for a family of diffusion processes $X^{1}, \ldots, X^{k}$ and for a closed set $B$. Then the formula

$$
F(x)=P_{x} \sum_{q \in Q} \tilde{Z}_{q} f\left(x_{\tau_{q}}\right)
$$

gives a generalized solution of the Dirichlet problem for the equation

$$
D_{x^{1}}^{1} \ldots D_{x^{k}}^{k} F\left(x^{1}, \ldots, x^{k}\right)=0
$$

where the infinitesimal generator of $X^{i}$ acts on $F$ as a function of $x^{i}$ with the frozen values of the rest of components.

For two one-dimensional Brownian motions this equation takes the form

$$
\frac{\partial^{4} F\left(x^{1}, x^{2}\right)}{\left(\partial x^{1}\right)^{2}\left(\partial x^{2}\right)^{2}}=0
$$

It has been studied by Wolpert in connection with the prediction problem for the Brownian sheet.

1.6. Let $\Phi$ be the Gaussian field associated with one Markov process $X$. Theorem 1.2.1 establishes the Markov property of $\Phi$ on every pair $B, C$ subject to condition 1.2.A. We say that a pair $B, C$ is standard if it satisfies 1.2.A and if $B \cup C=E$. A random variable $F$ is called a splittable functional of $\Phi$ if, for every standard pair $B, C$, there exists a functional $F_{B}$ of $\Phi_{B}$ and a functional $F_{C}$ of $\Phi_{C}$ such that $F=F_{B}+F_{C}$.

Let $(\Omega, \mathcal{F}, P)$ be the probability space on which $\Phi$ is given and let $F$ be a splittable functional of $\Phi$. Then the formula

$$
P^{F}(A)=\int_{A} e^{F(\omega)} P(d \omega)
$$

defines a new measure on $(\Omega, \mathscr{F})$ with respect to which the random field $\Phi$ is not Gaussian but has the Markov property on each standard pair $B, C$. For this reason, it is important to find all splittable functionals of $\Phi$.

This can be done in terms of Gaussian fields $\Phi^{k}, k=0,1,2, \ldots$, associated with $k$ indistinguishable replicas $X^{1}, \ldots, X^{k}$ of the process $X$. The field $\Phi^{k}$ is indexed by symmetric measures on $E^{k}$ ( $E$ is the state space of the process $X$ ). Relying on a theorem of Kakutani-Ito-Segal ${ }^{6}$, we establish a one-to-one correspondence between square-integrable functionals $F$ of $\Phi$ and sequences $Y_{0}, Y_{1}, \ldots, Y_{k}, \ldots$, where $Y_{k}$ is an element of the Hilbert space linearly generated by the field $\Phi^{k}$. To specify sequences corresponding to

\footnotetext{
${ }^{6}$ We give a proof of this theorem in the Appendix.
} 
splittable functionals, we denote by $H^{k}(B)$ the Hilbert space linearly generated by the values of $\Phi^{k}$ on the rectangle $B^{k}$ and we say that $Y$ is a tight functional of $\Phi^{k}$ if $Y$ belongs to $H^{k}(B)+H^{k}(C)$ for all standard pairs $B, C$. $A$ square-integrable functional $F$ of $\Phi$ is splittable if and only if the corresponding sequence $Y_{k}$ consists of tight functionals.

We have reduced the original problem to the problem of describing all tight functionals of the field $\Phi^{k}$. Let us say that $x, y \in E$ are neighbours if, for every standard pair $B, C$, either $x, y \in B$ or $x, y \in C$. Put $x=\left(x^{1}, \ldots, x^{k}\right)$ $\in D$ if all pairs $x^{i}, x^{j}$ are neighbours. All elements of the Hilbert space linearly generated by the values of $\Phi$ on $D$ are tight. The problem of describing all tight functionals remains open. (For the Nelson free field and some other stationary fields on Euclidean spaces, a related class of functionals has been recently studied in [4].)

1.7. The results presented in Subsections $1.2-1.4$ are based on a probabilistic representation of the covariance function $\langle\mu, \nu\rangle$.

Let $\zeta^{i}$ be the life time of the process $X^{i}$ and let $\zeta=\left(\zeta^{1}, \ldots, \zeta^{k}\right)$. In the case when $\nu(d x)=\rho(x) m(d x)$, we have

$$
\langle\mu, \nu\rangle=P_{\mu} \int_{0}^{\zeta} \rho\left(x_{t}\right) d t .
$$

The formula

$$
A(\omega, C)=\int_{C} \rho\left(x_{t}\right) 1_{t<5} d t
$$

determines, for every $\omega \in \Omega$, a measure $A(\omega, \cdot)$ on $T$ concentrated on the set $\{t: 0<t<\zeta\}$ with the following property:

1.7.A. For all $s<u \in T, A(\cdot,(s, u))$ is a functional of $x_{t}, s<t<u$.

A random measure with this property is called an additive functional of $X$. It turns out that, for every $\nu \in \mathscr{N}$, there exists an additive functional $A_{\nu}$ of $X$ such that

$$
\langle\mu, \nu\rangle=P_{\mu} A_{\nu}(T) .
$$

Hence

$$
E \varphi_{\mu} \varphi_{\nu}=P_{\mu} A_{\nu}(T) .
$$

Formula (1.16) makes it possible to use paths of Markov processes $X^{1}, \ldots, X^{k}$ for investigation of the associated Gaussian field $\Phi$.

Additive functionals of one Markov process have been studied by many authors. We refer to [6] and [2] for earlier history and to [9] and [11] for recent developments. In the case of several processes, the first nontrivial examples of additive functionals were investigated by Wolpert [30], [31]. A general theory is developed in [10].

1.8. We shall use the following notations. Suppose that $F$ is a real-valued function in a measure space $(\Omega, \mathscr{F}, P)$. We write $F \in \mathscr{F}$ if $F$ is measurable with respect to $\mathscr{F}$. We say that a set $C \subset \Omega$ is $P$-negligible if there exists a set $\Omega_{1} \in \mathscr{F}$ such that $C \subset \Omega_{1}$ and $P\left(\Omega_{1}\right)=0$. We write $F \in \mathscr{F}$ a.s. $P$ if $F$ coincides with a function $F_{1} \in \mathscr{F}$ outside a $P$-negligible set. Let $Y_{s}(\omega)$ and $Z_{s}(\omega)$ be two functions on $(\Omega, F, P)$ depending on a parameter $s \in S$. We say 
that $Y$ and $Z$ are $P$-indistinguishable if $Y_{s}(\omega)=Z_{s}(\omega)$ for all $s \in S$ outside a $P$-negligible set.

1.9. The author is indebted to L. Gross and R. Wolpert for stimulating discussions. $\mathrm{He}$ is especially grateful to $\mathbf{R}$. Vanderbei who participated actively in investigation of the prediction problem presented in $\$ 7$ and who contributed significantly to understanding of the subject. He also suggested a number of corrections and improvements to the manuscript.

\section{Gaussian random fields.}

2.1. Normal distributions form a class of measures on the Euclidean space $R^{n}$ which is invariant under all linear transformations

$$
y_{k}=\sum_{j} c_{k j} x_{j}+a_{k}, \quad k=1, \ldots, n
$$

and which contains the measure

$$
\mu(B)=(2 \pi)^{-n / 2} \int_{B} e^{-\frac{1}{2}\left(x_{1}^{2}+\cdots+x_{n}^{2}\right)} d x_{1} \ldots d x_{n} .
$$

If $X_{1}, \ldots, X_{n}$ are random variables with the probability distribution (2.2) and if $Y_{1}, \ldots, Y_{n}$ are connected with them by the transformation (2.1), then

$$
E Y_{k}=a_{k}, \quad E Y_{k} Y_{l}=\sigma_{k l}
$$

where

$$
\sigma_{k l}=\sum_{j} c_{k j} c_{l j}
$$

Obviously the matrix $\sigma_{k l}$ is positive semidefinite, i.e.,

$$
\sum_{k, l} \sigma_{k l} \lambda_{k} \lambda_{l}>0
$$

for all real numbers $\lambda_{1}, \ldots, \lambda_{n}$. On the other hand, every positive semidefinite matrix $\sigma_{k l}$ can be represented in the form (2.4). Therefore, to every vector $a_{k}$ and every positive semidefinite matrix $\sigma_{k l}$ there corresponds one and only one normal distribution satisfying conditions (2.3).

A random field $\Phi$ is a collection of random variables $\varphi_{s}, s \in S$ on a probability space $(\Omega, \mathcal{F}, P)$ indexed by elements of an arbitrary set $S$. A random field $\Phi$ is Gaussian if, for every $s_{1}, \ldots, s_{n} \in S$, the joint probability distribution of $\varphi_{s}, \ldots, \varphi_{s_{n}}$ is normal. These distributions are completely determined if $E \varphi_{s}$ and $E \varphi_{s} \varphi_{t}$ are given for all $s, t \in S$. Without any loss of generality, we can assume that $E \varphi_{s}=0$. The function $b(s, t)=E \varphi_{s} \varphi_{t}$ is called the covariance function. A function $b(s, t)$ is the covariance function of a Gaussian random field if and only if it is positive semidefinite, i.e., the matrix $\sigma_{k l}=b\left(s_{k}, s_{l}\right)$ is positive semidefinite for all $s_{1}, \ldots, s_{n} \in S, n=1,2, \ldots$, (see e.g. [17, Chapter 9, 88]).

2.2. To every subset $U$ of $S$ there corresponds a subfield $\Phi_{U}=\left\{\varphi_{u}\right.$, $u \in U$ \} of the field $\Phi$. Let $\mathscr{F}_{U}$ be the minimal $\sigma$-algebra in $\Omega$ with respect to which all functions $\varphi_{u}, u \in U$ are measurable. We denote by $E\left(Y \mid \Phi_{U}\right)$ the conditional mathematical expectation of $Y$ given $\mathscr{F}_{U}$.

A function $Z$ on $\Omega$ is called a functional of $\Phi_{U}$ if $Z \in \mathscr{F}_{U}$ a.s. $P$. Square integrable functionals of $\Phi_{U}$ form a subspace $L(U)$ of the Hilbert space 
$L^{2}(\Omega, \mathscr{F}, P)$. For every $Y \in L^{2}(\Omega, \mathscr{F}, P)$, the expectation $E\left(Y \mid \Phi_{U}\right)$ coincides with the orthogonal projection of $Y$ on $L(U)$. This is the best estimate of $Y$ by a functional of $\Phi_{U}$.

Let $H(U)$ be the minimal subspace of the Hilbert space $L(U)$ which contains $\varphi_{u}, u \in U$. We say that $H(U)$ is linearly generated by $\Phi_{U}$ and we call elements of $H(U)$ linear functionals of $\Phi_{U}$. If $Y$ is a linear functional of a Gaussian field $\Phi$, then for every subfield $\Phi_{U}$, the expectation $E\left(Y \mid \Phi_{U}\right)$ belongs to $H(U)$ and therefore is the orthogonal projection of $Y$ on $H(U)$.

2.3. Let $\Phi_{S_{S_{1}}}, \Phi_{S_{1}}, \Phi_{S_{2}}$ be subfields of a field $\Phi$. We say that $\Phi_{S_{1}}$ and $\Phi_{S_{2}}$ are conditionally independent given $\Phi_{S_{0}}$ if:

2.3.A. For every $Y_{1} \in L\left(S_{1}\right), Y_{2} \in L\left(S_{2}\right)$,

$$
E\left\{Y_{1} Y_{2} \mid \Phi_{S_{0}}\right\}=E\left\{Y_{1} \mid \Phi_{S_{0}}\right\} E\left\{Y_{2} \mid \Phi_{S_{0}}\right\} \text { a.s. } P \text {. }
$$

Put $S_{01}=S_{0} \cup S_{1}, S_{02}=S_{0} \cup S_{2}$. The condition 2.3.A is equivalent to

2.3.B. For every $Y \in L\left(S_{01}\right)$,

$$
E\left(Y \mid \Phi_{S_{02}}\right) \in L\left(S_{0}\right) \text {. }
$$

For Gaussian fields, conditions 2.3.A, B are equivalent to each of the following two assumptions.

2.3.C. The orthogonal projection of $Y \in H\left(S_{01}\right)$ on the space $H\left(S_{02}\right)$ belongs to $H\left(S_{0}\right)$.

2.3.D. $H\left(S_{01}\right) \odot H\left(S_{0}\right)$ is orthogonal to $H\left(S_{02}\right) \odot H\left(S_{0}\right)$. (Here $H \odot H^{\prime}$ means the orthogonal complement of $H^{\prime}$ in $H$.)

\section{Gaussian fields associated with Markov transition densities.}

3.1. Let $(E, \mathscr{B}, m)$ be a measure space and let $T_{+}=(0, \infty)$ be the open positive half-line. A Markov transition density is a positive function $p_{t}(x, y)$, $t \in T_{+}, x, y \in E$ with the following properties:

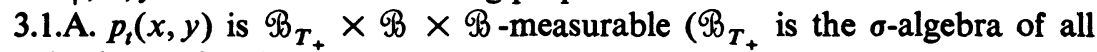
Borel subsets of $\left.T_{+}\right)$.

3.1.B. $\int_{E} p_{s}(x, y) m(d y) p_{t}(y, z)=p_{s+t}(x, z)$ for all $s, t \in T_{+}, x, z \in E$.

3.1.C. $\int_{E} p_{t}(x, y) m(d y)<1$ for all $t \in T_{+}, x \in E$.

We consider only densities which satisfy the additional conditions:

3.1.D. If $x \neq x^{\prime}$, then $p_{t}(x, y) \neq p_{t}\left(x^{\prime}, y\right)$ for some $t, y$.

3.1.E. (Symmetry) $p_{t}(x, y)=p_{t}(y, x)$ for all $t, x, y$.

To every transition density there corresponds a Markov process with a stationary transition function

$$
p_{t}(x, B)=\int_{B} p_{t}(x, y) m(d y)
$$

(see the definition in $\$ 4)$.

If $p_{t}(x, y)$ is a symmetric transition density, then so is $p_{t}^{\lambda}(x, y)=$ $e^{-\lambda p_{t}} p_{t}(x, y)$ for every $\lambda \in T_{+}$. We say that $p^{\lambda}$ is obtained from $p$ by $\lambda$-killing. Examples of symmetric transition densities are given in Subsection 1.2.

To every transition density there corresponds its Green function defined by formula (1.2). This function can be infinite identically. However the Green function corresponding to a killed density $p_{t}^{\lambda}(x, y)$ has the following property: for every $x, g(x, y)<\infty$ for $m$-almost all $y$. 
3.2. It follows from 3.1.B and 3.1.E that

$$
\sum_{1}^{n} g\left(x_{k}, x_{l}\right) c_{k} c_{l}=\int_{T_{+}} d t \int_{E} f(t, z)^{2} m(d z)>0
$$

where

$$
f(t, z)=\sum_{1}^{n} c_{k} p_{t / 2}\left(x_{k}, z\right) .
$$

Hence if $g$ is finite, it is positive semidefinite, and there exists a Gaussian random field $\Phi=\left\{\varphi_{x}, x \in E\right\}$ with the covariance function $g$. To every Green function there corresponds a positive semidefinite function $\langle\mu, \nu\rangle$ on the space $\mathscr{N}=\{\mu:\langle\mu, \mu\rangle<\infty\}$ defined by the formula (1.4). Thus there exists a Gaussian field $\Phi=\left\{\varphi_{\mu}, \mu \in \mathscr{T}\right\}$ satisfying the condition (1.5). We call $\Phi$ the Gaussian field associated with $p_{t}(x, y)$ (or with the Markov process $X$ corresponding to $p$ ).

Applying the same arguments to the function $g(x, y)$ defined by (1.6), we prove the existence of a Gaussian field associated with a family of symmetric transition densities.

3.3. We consider a metric in $\Re$ defined by the formula

$$
d(\mu, \nu)=(\langle\mu, \mu\rangle+\langle\nu, \nu\rangle-2\langle\mu, \nu\rangle)^{1 / 2} \text {. }
$$

LEMMA 3.1. Let $\mu \in \mathfrak{R}$ and let

$$
a^{\delta}(x)=\int_{E} p_{\delta}(x, y) \mu(d y), \quad \mu_{\delta}(d x)=a^{8}(x) m(d x) .
$$

Then $\mu_{8}$ belongs to $\Re$ and

$$
d\left(\mu_{\delta}, \mu\right) \rightarrow 0 \text { as } \delta \rightarrow 0 .
$$

Proof. Put

$$
\begin{gathered}
g^{i}\left(s^{i}, x^{i}, y^{i}\right)=\int_{s^{i}}^{\infty} p_{t}^{i}\left(x^{i}, y^{i}\right) d t, \quad i=1,2, \ldots, k, \\
g(s, x, y)=g^{1}\left(s^{1}, x^{1}, y^{1}\right) \ldots g^{k}\left(s^{k}, x^{k}, y^{k}\right) \\
\text { for } s=\left(s^{1}, \ldots, s^{k}\right), x=\left(x^{1}, \ldots, x^{k}\right), y=\left(y^{1}, \ldots, y^{k}\right) . \text { Let } \\
\langle\mu, \nu\rangle_{s}=\int_{E \times E} \mu(d x) g(s, x, y) \nu(d y) .
\end{gathered}
$$

It follows from (3.3) and 3.1.B that

$$
\left\langle\mu, \mu_{s}\right\rangle=\langle\mu, \mu\rangle_{s}, \quad\left\langle\mu_{s}, \mu_{t}\right\rangle=\langle\mu, \mu\rangle_{s+t}
$$

Therefore

$$
d\left(\mu_{\delta}, \mu\right)^{2}=\langle\mu, \mu\rangle+\langle\mu, \mu\rangle_{2 \delta}-2\langle\mu, \mu\rangle_{\delta} \rightarrow 0 \text { as } \delta \rightarrow 0 .
$$

LEMMA 3.1 implies that measures absolutely continuous with respect to $m$ form in $\mathfrak{N}$ an everywhere dense subset $\mathfrak{K}^{0}$. Note that finite measures are also everywhere dense in $\Re$. Indeed, if $\mu \in \mathfrak{R}$, then there exists a sequence $E_{n} \uparrow E$ such that the restriction $\mu_{n}$ of $\mu$ to $E_{n}$ is finite. We have

$$
d\left(\mu_{n}, \mu\right)^{2}=\int_{E_{x}^{c} \times E_{n}^{c}} \mu(d x) g(x, y) \mu(d y) \rightarrow 0 \text { as } n \rightarrow \infty \text {. }
$$




\section{Markov processes.}

4.1. To specify a Markov process with a state space $(E, \mathscr{B})$ and a sample space $(\Omega, \mathscr{F})$, we define

(i) a mapping $\zeta(\omega)$ of $\Omega$ into the extended half-line $(0,+\infty]$ (the life time);

(ii) for every $\omega \in \Omega, t \in\left[0, \zeta(\omega)\right.$ ), a point $x_{t}(\omega)$ of $E$ (the path corresponding to $\omega)$; $t$ );

(iii) for every $t>0$, a sub- $\sigma$-algebra $\mathscr{F}_{t}$ of $\mathscr{F}$ (events observable up to time

(iv) for every $x \in E$, a probability measure $P_{x}$ on $\mathcal{F}$ (the probability law corresponding to a starting point $x$ );

(v) for every $t>0$, a measurable transformation $\theta_{t}$ of $(\Omega, \mathcal{F})$ (the shift operator).

The collection $\zeta, x_{t}, \mathscr{F}_{t}, P_{x}, \theta_{t}$ defines a Markov process $X$ if:

4.1.A. $\mathscr{F}_{s} \subset \mathscr{F}_{t}$ for $s<t$, and $\left\{\omega: \zeta(\omega)>t, x_{t}(\omega) \in B\right\} \in \mathscr{F}_{t}$ for all $t>0$, $B \in \mathscr{B}$.

4.1.B. $P_{x}\left(x_{0}=x\right)=1$.

4.1.C. For every $C \in \mathscr{F}$, the function $f(x)=P_{x}(C)$ is $\mathscr{B}$-measurable.

4.1.D. $\theta_{t} \zeta(\omega)=\zeta(\omega)-t$ for $t<\zeta(\omega)$,

$$
\theta_{t} x_{s}(\omega)=x_{t+s}(\omega) \text { for } t+s<\zeta(\omega) \text {. }
$$

4.1.E. (MARKOV PROPERTY). For all $x \in E, t>0$ and all positive $Y \in \mathscr{F}_{t}$, $Z \in \mathcal{F}$,

$$
P_{x}\left(Y 1_{\zeta>t} \theta_{t} Z\right)=P_{x}\left(Y 1_{\zeta>t} P_{x_{i}} Z\right)
$$

To every measure $\mu$ on $(E, \mathscr{B})$, there corresponds a measure $P_{\mu}$ on $(\Omega, \mathscr{F})$ defined by the formula

$$
P_{\mu}(C)=\int_{E} \mu(d x) P_{x}(C)
$$

We say that a set $\Omega_{0}$ is negligible if it is $P_{\mu}$-negligible for all $\sigma$-finite measures $\mu$ and we write $Y \in \mathscr{F}$ a.s. if $Y \in \mathscr{F}$ a.s. $P_{\mu}$ for every $\mu$.

Formula (4.1) holds for every measure $P_{\mu}$ and all positive $Y, Z$ such that $Y \in \mathscr{F}_{t}$ a.s., $Z \in \mathcal{F}$ a.s.

4.2. In this paper we assume that the state space $(E, \mathscr{B})$ is a standard Borel space i.e. it is isomorphic to a Borel subset of a Euclidean space. Under this assumption, to every Markov transition density $p_{t}(x, y)$ on $(E, \mathscr{B})$ there corresponds a Markov process $X$ such that

$$
P_{x}\left(x_{t} \in B\right)=p_{t}(x, B), \quad t>0, x \in E, B \in \mathscr{B},
$$

where $p_{t}(x, B)$ is defined by (3.1). We call $X$ a symmetric Markov process if $p_{t}(x, y)$ satisfies the symmetry condition 3.1.E and condition 3.1.D. ${ }^{8}$

${ }^{7}$ This is true for every Markov transition function $p_{t}(x, B)$ independently of the representation (3.1) (see the definition of a Markov transition function, for example in [6, Chapter 21).

${ }^{8} \mathrm{~A}$ wider class of symmetric Markov processes is studied in the books of Silverstein [27] and Fukushima [11]: the existence of the density $p_{t}(x, y)$ is not assumed and the symmetry is required in terms of the transition function: $p_{t}(x, d y) m(d x)=p_{t}(y, d x) m(d y)$. On the other hand, both authors impose stronger regularity conditions than condition 4.2.A. 
Put

$$
\begin{aligned}
P_{\downarrow} f(x) & =\int_{E} p_{t}(x, d y) f(y), \\
G_{\lambda} f(x) & =\int_{0}^{\infty} e^{-\lambda t} P_{\downarrow} f(x) d t .
\end{aligned}
$$

We say that a Markov process $X$ is right if the following condition is satisfied.

4.2.A. For every positive $f \in \mathscr{B}, \lambda \geqslant 0$ and every probability measure $\mu$, the function

$$
Y_{t}(\omega)=G_{\lambda} f\left(x_{t}(\omega)\right) \quad \text { for } t \in[0, \zeta(\omega)), \quad Y_{t}(\omega)=0 \text { for } t>\zeta(\omega)(4.6)
$$

is $\boldsymbol{P}_{\mu}$-indistinguishable from a right-continuous function.

(This is a slight modification of "hypothéses droites" of Meyer. Right processes are treated in detail in [12] and [8]. They include the class of standard processes studied in [2] and [6].)

We call a transition density $p_{t}(x, y)$ regular if there exists a right Markov process $X$ satisfying (4.2) and (3.1). All densities considered in the Introduction are regular. Generally, $p$ is regular if $E$ is a locally compact separable metric space, $\mathscr{B}$ is the collection of all Borel subsets of $E$ and the transformations $P_{t}$ defined by (4.4) preserves the space $\hat{C}$ of continuous functions tending to 0 at infinity (see e.g. [6, Theorem 3.14]). This criterion is applicable to diffusions on differentiable manifolds for which $p$ is the fundamental solution of a certain parabolic differential equation.

There exists a wide class of random variables $\tilde{\zeta}<\zeta$ such that the reduction of the life time to $\tilde{\zeta}$ does not destroy the Markov character of the process (see [6, Chapter 10]). Under broad conditions, this transformation preserves both symmetry and the Property 4.2.A. This is an important source of symmetric regular transition densities. In particular, the $\lambda$-killing considered in subsection 3.1 corresponds to $\tilde{\zeta}$ independent of the path and having the exponential distribution $P(\tilde{\zeta}>u)=e^{-\lambda u}$. Another possibility is to kill at the first exit time from a given set $B$.

4.3. Conditions 4.2.A and 3.1.D imply that for every $f \in \mathscr{B}$ and every $\sigma$-finite measure $\mu$ the function $f\left(x_{t}(\omega)\right)$ is $P_{\mu}$-indistinguishable from a $\mathscr{B}_{T} \times$ F-measurable function. Applying Fubini's theorem, we deduce from (4.3), (4.4), (4.5) the following useful formula

$$
G_{\lambda} f(x)=P_{x} \int_{0}^{\zeta} e^{-\lambda t} f\left(x_{t}\right) d t .
$$

Let us consider a function $\tau(\omega)$ with values in the interval $[0, \zeta(\omega))$ and, possibly, a value $+\infty$. It is called a stopping time if $\{\tau<t\} \in \mathscr{F}_{t}$ a.s. for every $t>0$.

We put $C \in \mathscr{F}_{\tau}$ if $C \cap\{\tau<t\} \in \mathscr{F}_{t}$ a.s. for all $t$. Intuitively, this means that $C$ is observable in time interval $[0, \tau+\varepsilon)$ for every positive $\varepsilon$.

We shall use the following properties of right processes proved in [12] and [8] (in the case of standard processes they are also proved in [2] and [6]).

4.3.A. (THE STRONG MARKOV PROPERTY.) If $\tau$ is a stopping time, then for each measure $\mu$ and all positive $Y \in \mathscr{F}_{\tau}, Z \in \mathcal{F}$ a.s.

$$
P_{\mu}\left(Y 1_{\tau<\infty} \theta_{\tau} Z\right)=P_{\mu}\left(Y 1_{\tau<\infty} P_{x_{r}} Z\right) \text {. }
$$


4.3.B. For every progressive ${ }^{9}$ function $F(t, \omega), \tau(\omega)=\inf \{t: F(t, \omega)>0\}$ is a stopping time. In particular, for every $B \in \mathscr{B}, \tau_{B}=\inf \left\{t: t>0, x_{t} \in B\right\}$ is a stopping time (it is called the first hitting time of $B$ ).

A point $x$ is called regular for $B$ if, for every $u>0, P_{x}\left\{x_{t} \in B\right.$ for some $0<t<u\}=1$. A set $B$ is $f$-closed (or finely closed) if it contains all is regular points. The set $\bar{B}$ consisting of all points of $B$ and all points regular for $B$ is called the $f$-closure of $B$.

4.3.C. For every $B \in \mathscr{B}$ and every $\sigma$-finite measure $\mu$

$$
P_{\mu}\left\{x_{\tau_{B}} \notin \bar{B}\right\}=0 \text { and } P_{\mu}\left\{x_{\tau_{B}} \neq x_{\tau_{\bar{B}}}\right\}=0 \text {. }
$$

4.3.D. For every $B \in \mathscr{B}$ and every $\mu$, there exists a sequence of $f$-closed sets $B_{n} \subset B$ such that $\tau_{B_{n}} \uparrow \tau_{B}$ a.s. $P_{\mu}$.

A positive function $h \in B$ is called excessive if $P_{t} h(x) \leqslant h(x)$ for all $t, x$ and $P_{t} h(x) \rightarrow h(x)$ as $t \rightarrow 0$.

4.3.E. If $h$ is an excessive function, then, for every pair of stopping times $\sigma<\tau$ and all $x$

$$
P_{x} h\left(x_{\sigma}\right) \geqslant P_{x} h\left(x_{\tau}\right) .
$$

4.4. Let a Markov process $X^{i}=\left(\zeta^{i}, x_{t^{\prime}}^{i}, \mathscr{F}_{t^{\prime}} i, P_{x^{i}}^{i}, \zeta^{i}\right)$ with a state space $\left(E^{i}, \mathscr{B}^{i}\right)$ and a sample space $\left(\Omega^{i}, \mathscr{F}^{i}\right)$ be given for every $i=1,2, \ldots, k$. We consider the product spaces

$$
\begin{gathered}
(E, \mathscr{B})=\left(E^{1}, \mathscr{B}^{1}\right) \times \cdots \times\left(E^{k}, \mathscr{B}^{k}\right), \\
(\Omega, \mathscr{F})=\left(\Omega^{1}, \mathscr{F}^{1}\right) \times \cdots \times\left(\Omega^{k}, \mathscr{F}^{k}\right), \quad T=[0, \infty) \times \cdots \times[0, \infty)
\end{gathered}
$$

and we put

$$
\begin{gathered}
x_{t}(\omega)=\left(x_{t^{1}}^{1}\left(\omega^{1}\right), \ldots, x_{t^{k}}^{k}\left(\omega^{k}\right)\right), \\
\mathscr{F}_{t}=\mathscr{F}_{t^{1}}^{1} \times \cdots \times \mathscr{F}_{t^{k}}^{k}, \quad P_{x}=P_{x^{1}}^{1} \times \cdots \times P_{x^{k}}^{k}, \\
\theta_{t}(\omega)=\left(\theta_{t^{1}} \omega^{1}, \ldots, \theta_{t^{k}} \omega^{k}\right)
\end{gathered}
$$

for $\omega=\left(\omega^{1}, \ldots, \omega^{k}\right) \in \Omega, t=\left(t^{1}, \ldots, t^{k}\right) \in T, x=\left(x^{1}, \ldots, x^{k}\right) \in E$. The collection $\left(\zeta, x_{t}, F_{t}, P_{x}, \theta_{t}\right)$ describes a family $X$ of noninteracting Markov processes.

We consider the partial ordering of $T$ defined in subsection 1.3. Every two elements $s<u \in T$ determine a finite open interval $T_{u}^{s}=\{t: s<t<u\}$. We denote by $C+t$ the translation of $C$ through $t$ i.e. the set of all sums $c+t$, $c \in C$, and we put $T^{t}=T+t=\{u: t<u\}$.

All properties 4.1.A through $\mathrm{E}$ hold, the definitions of measures $\boldsymbol{P}_{\mu}$, stopping times $\tau$ and corresponding $\sigma$-algebras $F_{\tau}$ are valid. The strong Markov property 4.3.A holds if all processes $X^{1}, \ldots, X^{k}$ are right.

Note that, if $\tau^{i}$ is a stopping time for $X^{i}$, then $\tau=\left(\tau^{1}, \ldots, \tau^{k}\right)$ is a stopping time for $X$ and $\mathscr{F}_{\tau}=\mathscr{F}_{\tau^{1}} \times \cdots \times \mathscr{F}_{\tau^{k}}$.

${ }^{9} \mathrm{~A}$ real-valued function $F(t, \omega)$ is strictly progressive if, for every $u>0$, its restriction to the set $[0, u] \times \Omega$ is measurable with respect to $\mathscr{B}_{u} \times \mathscr{F}_{u}$ where $\mathscr{B}_{u}$ is the Borel $\sigma$-algebra on the interval $[0, u]$. A function $\mathcal{F}$ is progressive if, for every $\sigma$-finite measure $\mu, F$ is $P_{\mu}$-indistinguishable from a strictly progressive function. 
The definitions of regular points and $f$-closure do not need any modifications. We just remark that the $f$-closure of a rectangle $B=B^{1} \times \cdots \times B^{k}$ is the rectangle $\bar{B}=\bar{B}^{1} \times \cdots \times \bar{B}^{k}$ where $\bar{B}^{i}$ is the $f$-closure of $B^{i}$ relative to $X^{i}$.

\section{Additive functionals.}

5.1. Let $X$ be a family of noninteracting Markov processes. Additive functionals of $X$ were introduced in subsection 1.7. The precise meaning of the condition 1.7.A is as follows

5.1.A. Let $\mathscr{F}_{u}{ }^{s}$ be the minimal $\sigma$-algebra in $\Omega$ containing all sets $\{\omega$ : $\left.x_{t}(\omega) \in B\right\}, t \in T_{u}^{s}, B \in \mathscr{B}$. For all $s<u \in T$,

$$
A\left(\cdot, T_{u}^{s}\right) \in \mathscr{F}_{u}^{s} \text { a.s. }
$$

Our principal tool is the following theorem.

THEOREM 5.1. Let $X$ be a family of noninteracting symmetric right processes. To every measure $\nu \in M$ there corresponds an additive functional $A_{\nu}$ of $X$ with the properties

5.1.A. There exists a negligible set $\Omega_{0}$ such that for all $\omega \notin \Omega_{0}$,

(i) $A_{v}\left(\omega, T_{u}^{s}\right)$ is finite for all $0<s<u \in T$ and is continuous in $s$ and $t$,

(ii) $A_{v}\left(\theta_{t} \omega, T_{u}^{s}\right)=A_{v}\left(\omega, T_{u+t}^{s+t}\right)$ for all $s<u \in T$ and all $t \in T$.

5.1.B. For every positive $f \in \mathscr{B}_{T} \times \mathscr{B}$ and every measure $\mu$

$$
P_{\mu} \int_{T} f\left(t, x_{t}\right) A_{\nu}(d t)=\int_{T} d t \int_{E} \mu(d x) \int_{E} p_{t}(x, y) f(t, y) \nu(d y) .
$$

5.1.C. For every $\nu \in \mathfrak{N}$ and all $0<s<u \in T$

$$
P_{m}\left[A_{\nu}\left(T_{u}^{s}\right)-\int_{s}^{u} a^{\delta}\left(x_{t}\right) d t\right]^{2} \rightarrow 0 \text { as } \delta \rightarrow 0
$$

where $a^{8}(x)$ is defined by (3.3).

This theorem is proved in [10]. (If the family $X$ consists of one process, the proof is easy to get from the results of Chapter 5 of Fukushima's book [9]. However the method of [11] does not work in the general case.)

5.2. Formula (1.15) follows from 5.2.B with $f(t, x)=1$. We prove a few other implications of Theorem 5.1 which we use in the next sections.

5.2.A. If $\nu$ is concentrated on $B$ and if $Y_{t}(\omega)=0$ for all $t, \omega$ such that $x_{t}(\omega) \in B$, then $\int_{T} Y_{t}(\omega) A_{\nu}(d t)=0$ a.s. $P_{\mu}$ for all measures $\mu$.

5.2.B. For every stopping time $\tau$, positive $Z \in \mathscr{F}_{\tau}$ and $C \in \mathscr{B}_{T}$

$$
P_{\mu} Z A_{\nu}(\tau+C)=P_{\mu} Z P_{x_{\gamma}} A_{\nu}(C) .^{10}
$$

5.2.C. Let $T(s)=\left\{t: t \in T, t^{i}=s^{i}\right.$ for some $\left.i\right\}$. For every $T$-valued random variable $\sigma$ and for all $\mu, \nu \in \Re$

$$
A_{\nu}(T(\sigma))=0 \text { a.s. } P_{\mu} \text {. }
$$

To prove 5.2.A, we note that $1_{Y, \neq 0}<1_{E \backslash B}\left(x_{t}\right)$ and we apply 5.1.B to $f(t, x)=1_{E \backslash B}(x)$. The statement 5.2.B follows from 5.1.A (ii) and 4.3.A. The property 5.3.C is an immediate implication of 5.1.A (i).

\footnotetext{
${ }^{10}$ The functions $A_{y}(\tau+C)$ and $P_{x_{r}} A_{y}(C)$ are not defined if $\tau=\infty$. We apply the following general rule: if a function under the integral sign is not defined on a set $\Omega^{\prime}$, put it equal to 0 .
} 
6. Markov property of random fields associated with Markov processes.

6.1. In this section we prove Theorems 1.2.1 and 1.2.2 with the following rigorous interpretation of condition 1.2.A:

6.1.A. For every $u>0, x \in B, y \in C$

$$
\begin{aligned}
P_{x}\left\{x_{t} \notin B \cap C\right. & \text { for } \left.0<t<u \text { and } x_{u} \in C\right\} \\
& =P_{y}\left\{x_{t} \notin B \cap C \text { for all } 0<t<u \text { and } x_{u} \in B\right\}=0 .
\end{aligned}
$$

It follows from 6.1.A that

6.1.B. For every measure $\mu, \tau_{B \cap C}=\max \left(\tau_{B}, \tau_{C}\right)$ a.s. $P_{\mu}$.

To prove this, we put $\tau=\min \left(\tau_{B}, \tau_{C}\right), \tau^{\prime}=\max \left(\tau_{B}, \tau_{C}\right)$ and we remark that $\theta_{\tau} \tau_{B}=\tau_{B}-\tau, \theta_{\tau} \tau_{C}=\tau_{C}-\tau$. By the strong Markov property 4.3.A,

$$
P_{\mu}\left\{\tau_{B \cap C}>\tau^{\prime}\right\}=P_{\mu} \theta_{\tau}\left(\tau_{B \cap C}>\tau^{\prime}\right)=P_{\mu} P_{x}\left(\tau_{B \cap C}>\tau^{\prime}\right) \text {. }
$$

By 4.3.C, $x_{\tau}$ belongs to the f-closure of $B$ or $C$. By 6.1.A, in both cases $P_{x_{\tau}}\left(\tau_{B \cap C}>\tau^{\prime}\right)=0$. Hence $\tau_{B \cap C}<\tau^{\prime}$ a.s. $P_{\mu}$ which, obviously, implies 6.1.B.

We deduce Theorem 1.2.1 from Theorem 1.2.2 which we prove first.

6.2. Since the field $\Phi$ is Gaussian, Theorem 1.2.2 is, according to subsection 2.2, equivalent to the following statement: If measures $\mu$ and $\mu_{B}$ are connected by the formula

$$
\mu_{B}(C)=P_{\mu}\left(x_{\tau} \in C\right)
$$

where $\tau$ is the first hitting time of $B$, then $\varphi_{\mu_{B}}$ is the orthogonal projection of $\varphi_{\mu}$ on the space $H(B)$ linearly generated by $\Phi_{B}=\left\{\varphi_{\nu}, \nu \in \mathfrak{R}(B)\right\}$.

In order to prove this statement, we need to check that

$$
\begin{gathered}
E \varphi_{\mu} \varphi_{\nu}=E \varphi_{\mu_{s}} \varphi_{\nu} \quad \text { for all } \nu \in \mathfrak{T}(B) ; \\
\varphi_{\mu_{s}} \in H(B) .
\end{gathered}
$$

By (1.16), the relation (6.2) is equivalent to

$$
P_{\mu} A_{\nu}(T)=P_{\mu_{s}} A_{\nu}(T) \text { for all } \nu \in \mathscr{T l}(B) \text {. }
$$

It follows from (6.1) that

$$
\mu_{B}(f)=P_{\mu} f\left(x_{\tau}\right)
$$

for every positive $f \in \mathscr{B}$. Taking $f(x)=P_{x} A_{\nu}(T)$ and using 5.2.B, we have

$$
P_{\mu_{s}} A_{\nu}(T)=P_{\mu} P_{x_{p}} A_{\nu}(T)=P_{\mu} A_{\nu}\left(T^{\tau}\right)
$$

where $T^{\tau}=\tau+T$. Since $\tau$ is the first hitting time of $B, P_{\mu} A_{\nu}\left(T \backslash T^{\tau}\right)=0$ for $\nu \in \mathscr{N}(B)$ by 5.2.A. The relation (6.4) follows from (6.6).

Now we prove (6.3). Put $h(x)=\int_{E} g(x, y) \nu(d y)$. It follows from 3.1.B that

$$
P_{x} h\left(x_{s}\right)=\int_{E} g(s, x, y) \nu(d y)
$$

where $g(s, x, y)$ is defined by (3.4). Hence $h$ is an excessive function and, by 4.3.E, $P_{x} h\left(x_{\tau}\right)<h(x)$. Using (1.4) and (6.5), we get

$$
\left\langle\mu_{B}, \nu\right\rangle=\mu_{B}(h)=P_{\mu} h\left(x_{\tau}\right)\langle\mu(h)=\langle\mu, \nu\rangle .
$$

Suppose that $\mu \in \mathfrak{N}$. Then

$$
\left\langle\mu_{B}, \mu_{B}\right\rangle\left\langle\left\langle\mu, \mu_{B}\right\rangle\langle\langle\mu, \mu\rangle<\infty\right.
$$


and $\mu_{B} \in \Re$. If $B$ is $f$-closed, then $\mu_{B} \in \mathfrak{N}(B)$ by 4.3.C. If $B$ is an arbitrary set of $\mathscr{B}$, then, by 4.3.D, $\tau_{B_{n}} \uparrow \tau_{B}=\tau$ a.s. $P_{\mu}$ for a sequence of $f$-closed sets $B_{n} \subset B$. Put $Z_{n}=\varphi_{\mu_{B_{n}}}$. It follows from (1.16) and (6.6) that

$$
\lim E Z_{n} \varphi_{\nu}=E \varphi_{\mu_{B}} \varphi_{\nu} \text { for all } \nu \in \Re
$$

Besides $E Z_{n}^{2} \leqslant\langle\mu, \mu\rangle$. Hence

$$
\lim E Z_{n} Y=E \varphi_{\mu_{B}} Y \text { for every } Y \in H(E) .
$$

Suppose that $Y$ is orthogonal to $H(B)$. Since $Z_{n} \in H(B)$, we have $E Z_{n} Y=0$ and hence $E \varphi_{\mu_{s}} Y=0$. This proves (6.3).

COROllary. If $\bar{B}$ is the $f$-closure of $B$, then $H(\bar{B})=H(B)$.

Indeed, by 4.3.C, $\tau_{B}=\tau_{\bar{B}}$ a.s. $P_{\mu}$, hence $\mu_{B}=\mu_{\bar{B}}$ and, by Theorem 1.2.2, the orthogonal projections of $\varphi_{\mu}$ on $H(B)$ and $H(\bar{B})$ coincide.

6.3. Let us prove Theorem 1.2.1. Denote by $\mu^{\prime}$ and $\mu^{\prime \prime}$ the restrictions of a measure $\mu$ to the sets $C^{\prime}=C \cap B$ and $C^{\prime \prime}=C \backslash C^{\prime}$. If $\mu \in \Re(C)$, then $\mu=\mu^{\prime}+\mu^{\prime \prime}$ and $\varphi_{\mu}=\varphi_{\mu^{\prime}}+\varphi_{\mu^{\prime \prime}}$. Since $\mu^{\prime} \in \Re(C \cap B)$, we have $E\left(\varphi_{\mu^{\prime}} \mid \Phi_{B}\right)$ $=\varphi_{\mu^{\prime}} \in H(C \cap B)$. By 6.1.B, $\tau_{B}=\tau_{B \cap C}$ a.s. $P_{\mu^{\prime \prime}}$. Hence $\mu_{B}^{\prime \prime}=\mu_{B \cap C}^{\prime \prime}$ and, by Theorem 1.1.2, $E\left(\varphi_{\mu^{\prime \prime}} \mid \Phi_{B}\right)=\varphi_{\mu_{B}^{\prime \prime}} \in H(B \cap C)$. Now the statement of Theorem 1.2.1 follows from 2.3.C.

6.4. In conclusion, we prove that the transformation (1.12) with a splittable $F$ preserves the Markov property on every standard pair $B, C$.

Put $Z=e^{F}, Z_{B}=e^{F_{B}}, Z_{C}=e^{F_{C}}$. The proof is based on the elementary identity

$$
P^{F}\left(Y \mid \Phi_{C}\right)=P\left(Y Z \mid \Phi_{C}\right) / P\left(Z \mid \Phi_{C}\right) \text { a.s. } P \text {. }
$$

Since $Z=Z_{B} Z_{C}$, this implies

$$
P^{F}\left(Y \mid \Phi_{C}\right)=P\left(Y Z_{B} \mid \Phi_{C}\right) / P\left(Z_{B} \mid \Phi_{C}\right) \text { a.s. } P \text {. }
$$

According to subsection 2.3, the Markov property of a random field on $B$, $C$, is equivalent to the property 2.3.B for $S_{1}=B, S_{2}=C, S_{0}=B \cap C$. It follows from (6.8) that 2.3.B holds for $P^{F}$ if it holds for $P$.

7. The prediction problem.

7.1. We start with the proof of Theorem 1.4.1. (Of course, condition 1.4.A means that $Z_{q} \in \mathscr{F}_{\tau_{q}}$.)

By (1.8) and (1.9), if $\hat{\mu}_{q}(|f|)<\infty$, then

$$
\left|\mu_{q}(f)\right|=\left|P_{\mu} \tilde{Z}_{q} f\left(x_{\tau_{q}}\right)\right|<P_{\mu}\left|\tilde{Z}_{q}\right|\left|f\left(x_{\tau_{q}}\right)\right|=\hat{\mu}_{q}(|f|) \text {. }
$$

By (1.5),

$$
E\left(\varphi_{\mu_{q}}\right)^{2}=\iint \mu_{q}(d x) g(x, y) \mu_{q}(d y)<\iint \hat{\mu}_{q}(d x) g(x, y) \hat{\mu}_{q}(d y)=\left\|\hat{\mu}_{q}\right\|^{2},
$$

and the series in (1.11) converges. Denote its sum by $Y$. Since $\mu_{q}$ is concentrated on $B$, we have $Y \in H(B)$ and we need only to prove that, for every $\nu \in M(B)$

$$
E Y \varphi_{\nu}=E \varphi_{\mu} \varphi_{\nu}
$$

By (1.16)

$$
E Y \varphi_{\nu}=\sum_{q} E \varphi_{\mu_{q}} \varphi_{\nu}=\sum_{q} P_{\mu_{q}} A_{\nu}(T)
$$


It follows from 5.2.B that, for every $\nu \in M$,

$$
P_{\mu_{q}} A_{\nu}(T)=P_{\mu} \tilde{Z}_{q} A_{\nu}\left(T^{\tau_{q}}\right)=P_{\mu} \int_{T} \tilde{Z}_{q} 1_{\tau_{q}<t} A_{\nu}(d t)
$$

and

$$
P_{\hat{\mu}_{q}} A_{\nu}(T)=P_{\mu} \int_{T}\left|\tilde{Z}_{q}\right| 1_{\tau_{q}<t} A_{\nu}(d t)
$$

We have

$$
P_{\hat{\mu}_{q}} A_{\nu}(T)=\left\langle\hat{\mu}_{q}, \nu\right\rangle \leqslant\left\|\hat{\mu}_{q}\right\|\|\nu\|,
$$

and, by (1.16), the series (7.5) converges. By the dominated convergence theorem, (7.4) implies that

$$
\sum_{q} P_{\mu_{q}} A_{\nu}(T)=P_{\mu} \int_{T} \sum_{\tau_{q}<t} \tilde{Z}_{q} A_{\nu}(d t) .
$$

Suppose that $\mu \in \mathscr{T}(B)$. It follows from 1.4.B and 5.2.A that the right side of (7.6) is equal to $P_{\mu} A_{\nu}(T)=E \varphi_{\mu} \varphi_{\nu}$, and (7.2) follows from (7.3) and (7.6).

7.2. Relying on Theorem 1.4.1, we investigate the prediction problem for finite unions of rectangles. Obviously, the solutions for sets $B$ and $C$ coincide if $H(B)=H(C)$. Suppose that $B$ is the union of rectangles $B_{1}, \ldots, B_{n}$ and $\bar{B}_{i}$ is the $f$-closure of $B_{i}$. We claim that $H(B)=H(\bar{B})$ where $\bar{B}=\bar{B}_{1}$ $\cup \cdots \cup \bar{B}_{n}$. The inclusion $H(B) \subset H(\bar{B})$ is evident. On the other hand, if $\mu \in \mathfrak{N}(\bar{B})$, then $\mu=\mu_{1}+\cdots+\mu_{n}$ where $\mu_{i} \in \mathfrak{N}\left(\bar{B}_{i}\right)$. By the corollary at the end of subsection $6.2, H\left(\bar{B}_{i}\right)=H\left(B_{i}\right)$. Hence $\varphi_{\mu} \in H\left(B_{i}\right) \subset H(B)$ and $\varphi_{\mu} \in H(B)$.

Due to this observation, we lose no generality considering only unions of $f$-closed rectangles.

7.3. For an $f$-closed rectangle $B$, a resolving system consists of one stopping time $\tau_{B}$ and one function $Z_{B}=1$. The next simplest example is a union of two rectangles.

THeOREM 7.1. Suppose that $B^{i}, C^{i}$ is a pair of $f$-closed sets subject to condition 6.1.A with respect to a process $X^{i}, i=1, \ldots, k . A$ resolving system for the union of rectangles $B=B^{1} \times \cdots \times B^{k}$ and $C=C^{1} \times \cdots \times C^{k}$ consists of three stopping times $\tau_{B}, \tau_{C}$ and $\tau_{B \cap C}$ with the corresponding functions $Z_{B}=Z_{C}=1, Z_{B \cap C}=-1$.

Proof. By 6.1.B, for every $i, \max \left(\tau_{B^{i}}^{i}, \tau_{C^{i}}^{i}\right)=\tau_{B^{i} \cap C^{i}}^{i}$. Therefore if $t>\tau_{B}$ and $t>\tau_{C}$, then $t>\tau_{B \cap C}$. If $x_{t} \in B$, then the set $\mathscr{I}_{t}$ either consists of one element $\tau_{B}$ or $\tau_{C}$ or it includes all three stopping times $\tau_{B}, \tau_{C}$ and $\tau_{B \cap C}$. The condition 1.4. $\mathrm{B}$ is satisfied in all cases. Condition 1.4.A is trivial.

COROLlaRY. Under the conditions of Theorem 7.1

$$
E\left(\varphi_{\mu} \mid \Phi_{B \cup C}\right)=E\left(\varphi_{\mu} \mid \Phi_{B}\right)+E\left(\varphi_{\mu} \mid \Phi_{C}\right)-E\left(\varphi_{\mu} \mid \Phi_{B \cap C}\right) .
$$

This implies the relations

$$
H(B \cup C)=H(B)+H(C), \quad H(B \cap C)=H(B) \cap H(C) .
$$

7.4. THEOREM 7.2. Let $B_{1}^{1} \supset \cdots \supset B_{n}^{1}$ be a decreasing sequence of $f$-closed sets for a process $X^{1}$, and $B_{1}^{2} \subset \cdots \subset B_{n}^{2}$ be an increasing sequence of $f$-closed 
sets for a process $X^{2}$. Denote by $\tau_{j}^{i}$ the first hitting time of $B_{j}^{i}$ by $X^{i}$. A resolving system for the set $B=\cup_{1}^{n} B_{j}^{1} \times B_{j}^{2}$ is given by the formulae

$$
\begin{aligned}
& \tau_{j}=\left(\tau_{j}^{1}, \tau_{j}^{2}\right), \quad j=1,2, \ldots, n, \\
& \tau_{j, j+1}=\left(\tau_{j+1}^{1}, \tau_{j}^{2}\right), \quad j=1, \ldots, n-1, \\
& Z_{1}=\ldots=Z_{n}=1, \quad Z_{12}=\ldots=Z_{n-1, n}=-1 .
\end{aligned}
$$

PRoof. If $x_{t} \in B$, then $\tau_{j} \in \mathscr{Z}_{t}$ at least for one $j$. Let $i$ be the smallest and $l$ be the largest value of $j$ for which $\tau_{j} \in \mathbb{Z}_{t}$. Since $\left.\tau_{1}^{1}\left\langle\cdots<\tau_{n}^{1}, \tau_{1}^{2}\right\rangle \cdots\right\rangle$ $\tau_{n}^{2}$, we have $\mathscr{Z}_{t}=\left\{\tau_{i}, \ldots, \tau_{l} ; \tau_{i, i+1}, \ldots, \tau_{l-1, l}\right\}$. Hence 4.1.B holds. Again 4.1.A is trivial.

COROLLARY. Under the conditions of Theorem 7.2, for every $\mu \in \mathfrak{N}$,

$$
E\left\{\varphi_{\mu} \mid \Phi_{B}\right\}=\varphi_{\mu_{s}}
$$

where

$$
\mu_{B}(f)=P_{\mu}\left[\sum_{j=1}^{n-1}\left[f\left(x_{\tau_{j}^{1}}^{1}, x_{\tau_{j}^{2}}^{2}\right)-f\left(x_{\tau_{j+1}^{1}}^{1}, x_{\tau_{j}^{2}}^{2}\right)\right]+f\left(x_{\tau_{n}^{1}}^{1}, x_{\tau_{n}^{2}}^{2}\right)\right] .
$$

7.5. Construction of resolving systems in a more general situation can be done using the following

THEOREM 7.3. Let $Q$ be the set of all vectors $q=\left(q^{1}, \ldots, q^{k}\right)$ with integral coordinates $q^{i}>0$. Let a stopping time

$$
\tau_{q}=\left(\tau_{q^{1}}, \ldots, \tau_{q^{k}}\right)
$$

be given for every $q \in Q$ and let

$$
0=\tau_{0}^{i}<\tau_{1}^{i}<\cdots<\tau_{j}^{i}<\cdots, \quad \lim _{j \rightarrow \infty} \tau_{j}^{i}=\zeta^{i} \text { or }+\infty \text { a.s. } P_{x}
$$

for every $i=1, \ldots, k$ and every $x \in E . P u t$

$$
I_{j}^{i}=\left\{t: \tau_{j}^{i}<t<\tau_{j+1}^{i}\right\}, \quad I_{q}=I_{q^{1}}^{1} \times \cdots \times I_{q^{k}}^{k}
$$

and suppose that

$$
\left\{x_{\tau_{q}} \notin B\right\} \subset\left\{x_{t} \notin B \text { for all } t \in I_{q}\right\} \text {. }
$$

Put $l<q$ if $l<q, l \neq q$. Define $Z_{q}$ by the recurrent formula

$$
Z_{q}=1_{B}\left(x_{\tau_{q}}\right)\left(1-\sum_{l<q} Z_{l} 1_{B}\left(x_{\tau_{l}}\right)\right) \text {. }
$$

Then $\left(\tau_{q}, Z_{q}\right)$ is a resolving system for $B$.

Proof. Again only 1.4.B needs verifying. If $x_{t} \in B$, then $t$ belongs to one and only one rectangle $I_{q}$ and, by (7.9) $x_{\tau_{q}} \in B$. It follows from (7.10) that

$$
\sum_{l<q} Z_{l} 1_{B}\left(x_{\tau_{l}}\right)=1
$$

For $t \in I_{q}, \tau_{l}<t$ if and only if $l<q$, and 1.4.B follows from (7.11).

7.6. The condition (7.9) is satisfied if $B$ is the union of rectangles $B=$ $B_{l^{1}}^{1} \times \cdots \times B_{l^{k}}^{k}$ and if, for every $i$ and $j$, 


$$
\left\{x_{\tau_{j}^{\prime}}^{i} \notin B_{j}^{i}\right\} \subset\left\{x_{t}^{i} \notin B_{j}^{i} \text { for all } t \in I_{j}^{i}\right\} .
$$

A sequence $\tau_{j}^{i}$ subject to conditions (7.12) can be defined in the following way. Fix $i$ and put $t \in \Lambda_{s}$ if $t>s$ and if there exists $j$ such that $x_{s}^{i} \notin B_{j}^{i}$ and $x_{t}^{i} \in B_{j}^{i}$. Let $F(s)=\inf \Lambda_{s}$ (or $+\infty$ if $\Lambda_{s}$ is empty). The sequence

$$
\tau_{0}^{i}=0, \quad \tau_{j+1}^{i}=F\left(\tau_{j}^{i}\right) \text { for } j=0,1,2, \ldots,
$$

satisfies (7.12). Using 4.3.B, it is easy to check that $\tau_{j}^{i}$ are stopping times for $X^{i}$. Hence (7.7) are stopping times for $X$.

7.7. The function $Z_{q}$ defined by formula (7.10) can be written down explicitly

$$
Z_{q}=\sum_{n=1}^{\infty}(-1)^{n-1} \sum_{l_{1}<\ldots<l_{n}=q} 1_{B}\left(x_{\tau_{1}}\right) \ldots 1_{B}\left(x_{\tau_{n}}\right) .
$$

This formula has a simple combinatorial meaning. A random set $\mathbb{Z}$ in $T$ is naturally associated with our problem. This is the set of all $\tau_{q}$ for which $X_{\tau_{q}} \in B$. Obviously $\mathcal{Z}$ is locally finite in the following sense: for every element of $\mathscr{Z}$, there exists only a finite number of smaller elements.

Suppose that $\mathcal{Q}$ is an arbitrary locally finite partially ordered set. We say that elements $a_{1}, \ldots, a_{n}$ of $\mathbb{Q}$ form $a$ chain with the end $a$ if $a_{1}<\cdots<a_{n}$ $=a$. Denote by $\kappa_{+}(a)$ and $\kappa_{-}(a)$ the numbers of chains with the end $a$ having, respectively, an even and an odd number of elements. Formula (7.14) means that

$$
Z_{q}=\kappa_{-}\left(\tau_{q}\right)-\kappa_{+}\left(\tau_{q}\right) \text { if } \tau_{q} \in \mathscr{Z}
$$

(We note that

$$
\kappa_{-}(a)-\kappa_{+}(a)=\sum_{b<a} \mu(b, a)
$$

where $\mu$ is the Möbius function of the set $\mathcal{Q}$ (see, e.g. [13, Chapter 2].)

7.8. Stopping times $\tau_{q}$ defined by (7.13) satisfy the following relations

$$
\tau_{l}+\theta_{\tau_{l}} \tau_{q}=\tau_{l+q}, \quad \theta_{\tau_{l}} x_{\tau_{q}}=x_{\tau_{l+q}}
$$

Let us introduce operators $P^{q}$ acting on measures by the formula

$$
\left(\mu \mathbf{P}^{q}\right)(f)=P_{\mu} f\left(x_{\tau_{q}}\right)
$$

and let $\mu^{B}$ be the restriction of the measure $\mu$ to the set $B$. Formulae (7.10) and (7.16) imply the following recurrent relations for the measures $\mu_{q}$ defined by (1.8)

$$
\mu_{q}=\left(\mu \mathrm{P}^{q}-\sum_{l<q} \mu_{l} \mathbf{P}^{q-l}\right)^{B}
$$

Using (1.11) and (7.18), we can evaluate $E\left(\varphi_{\mu} \mid \Phi_{B}\right)$ without computing $Z_{q}$.

7.9. A resolving system for the complement $B$ of a rectangle $C=$ $C^{1} \times \cdots \times C^{k}$ can be constructed in the following way.

For every subset $u$ of the set $1,2, \ldots, k$, denote by $B_{u}$ the rectangle $B_{u}^{1} \times \cdots \times B_{u}^{k}$ where $B_{u}^{i}=E^{i} \backslash C^{i}$ if $i \in u, B_{u}^{i}=E^{i}$ if $i \notin u$. Let $\tau_{u}$ be the first hitting time of $B_{u}$ and let $Z_{u}=(-1)^{|u|-1}$ where $|u|$ means the cardinality 
of the set $u$. A resolving system for $B$ is formed by $\left(\tau_{u}, Z_{u}\right)$ where $u$ runs over all nonempty subsets.

To prove this, we put $i \in v$ if $x_{t}^{i} \in E^{i} \backslash C^{i}$ for some $t$, and we note that the set $\mathcal{Z}$ of subsection 7.5 consists of all $\tau_{u}, u \subset v$. The expression for $Z_{u}$ follows from (7.10) or (7.15).

\section{Gaussian fields associated with indistinguishable replicas of a Markov process.}

8.1. Let us consider $k$ indistinguishable replicas of a Markov process $X$. Since all particles look identically, only symmetric functions of $\omega=$ $\left(\omega^{1}, \ldots, \omega^{k}\right)$ can be observed. The corresponding Gaussian field $\Phi^{k}$ is indexed by symmetric measures on $E^{k}$.

"Symmetric" means invariant with respect to the group $S$ of all permutations which acts on functions and measures on $E^{k}$ by formulae

$$
f^{s}(x)=f(s x), \quad \mu^{s}(B)=\mu\left(s^{-1} B\right)
$$

where $s x=\left(x^{s(1)}, \ldots, x^{s(k)}\right)$ for $x=\left(x^{1}, \ldots, x^{k}\right)$. Obviously $\mu^{s}(f)=\mu\left(f^{s}\right)$. Similar formulae hold for the space $\Omega^{k}$. Note that

$$
\left(\varphi_{\mu}\right)^{s^{-1}}=\varphi_{\mu}, \quad\left(P_{\mu}\right)^{s^{-1}}=P_{\mu} \text {. }
$$

For every class $K$ of functions or measures, we denote by $K_{S}$ the set of all symmetric elements of $K$.

To get the field $\Phi^{k}$, we consider the field $\varphi_{\mu}, \mu \in \mathfrak{T}$ associated with $X^{1}, \ldots, X^{k}$ and we restrict the index set to $\mathfrak{N}_{S}$.

8.2. The space $H_{S}(B)$ is linearly generated by $\Phi_{B}^{k}=\left\{\varphi_{\mu}, \mu \in \mathfrak{T}_{S}(B)\right\}$. Let $B_{S}$ be the intersection of $s^{-1} B$ for all $s \in S$. Evidently $\mathfrak{R}_{S}(B)=\mathscr{R}\left(B_{S}\right)$. Hence $H_{S}(B)=H_{S}\left(B_{S}\right)$ and the prediction problem for $\Phi^{k}$ should be examined only for symmetric sets $B$. In this case $E\left(\varphi_{\mu} \mid \Phi_{B}^{k}\right)$ is a symmetric function of $\omega$ and the expressions

$$
E\left(\varphi_{\mu} \mid \Phi_{B}^{k}\right)=\sum \varphi_{\mu_{q}}, \quad \mu_{q}(F)=P_{\mu} \tilde{Z}_{q} f\left(x_{\tau_{q}}\right)
$$

imply that

$$
\begin{aligned}
& E\left\{\varphi_{\mu} \mid \Phi_{B}^{k}\right\}=\sum \varphi_{\mu_{i}^{*},} \\
& \mu_{q}^{*}(f)=\frac{1}{k !} \sum_{s \in S} \mu_{q}^{s}(f)=\frac{1}{k !} P_{\mu} \sum_{s \in S} \tilde{Z}_{q}^{s^{-1}} f\left(x_{s(\tau)}\right)
\end{aligned}
$$

where $s(\tau)=\left(\tau^{s(1)}(s \omega), \ldots, \tau^{s(k)}(s \omega)\right)$ for $\tau=\left(\tau^{1}, \ldots, \tau^{k}\right)$.

The measures $\mu_{q}^{*}$ are symmetric and $\varphi_{\mu_{*}^{*}}$ belong to $H_{S}(B)$.

8.3. To every $B \in \mathscr{B}$, there corresponds a symmetric rectangle $B^{k}$. Let $\tau_{B}^{k}$ be the first hitting time of $B^{k}$. Put

$$
\mu_{B}^{k}(f)=P_{\mu} f\left(x_{\tau_{B}^{k}}\right), \quad H^{k}(B)=H_{S}\left(B^{k}\right) .
$$

By subsection 7.2, $\varphi_{\mu_{B}^{k}} \in H\left(B^{k}\right)$ and, since $\mu_{B}^{k}$ is symmetric, $\varphi_{\mu_{B}^{k}}$ belongs to $H^{k}(B)$.

Let $B, C$ be a standard pair for the process $X$ and let $N=B^{k} \cup C^{k}$. It follows from Theorem 7.1 that

$$
E\left(\varphi_{\mu} \mid \Phi_{N}^{k}\right)=\varphi_{\mu_{s}^{k}}+\varphi_{\mu_{c}^{k}}-\varphi_{\mu_{B}^{k} \cap}
$$


This formula can be used for investigating tight functionals of $\Phi^{k}$.

8.4. The statements in subsection 1.6 on the relation between the splittable functionals of the Gaussian field $\Phi$ associated with a Markov process $X$ and tight functionals of $\Phi^{k}$ follow immediately from the following theorem proved in the Appendix.

THEOREM 8.1. Let the transition density of $X$ satisfy the condition:

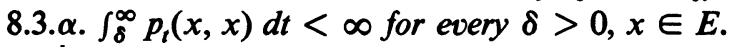

Let $\Phi^{k}$ be the Gaussian field associated with $k$ indistinguishable replicas of the process $X$ and let $\Phi=\Phi^{1}$. Then there exists, for every $k=0,1, \ldots, a$ mapping $\pi_{k}$ of $H^{k}=H^{k}(E)$ into the space $L_{\Phi}$ of all square-integrable functionals of $\Phi$ such that

8.3.A. $E \pi_{k}\left(Y_{1}\right) \pi_{l}\left(Y_{2}\right)=0$ if $k \neq l$,

$$
E \pi_{k}\left(Y_{1}\right) \pi_{k}\left(Y_{2}\right)=E Y_{1} Y_{2}
$$

8.3.B. Every $F \in L_{\Phi}$ has a unique representation $F=\sum_{k=0}^{\infty} \pi_{k}\left(Y_{k}\right), Y_{k} \in$ $H^{k}(E)$.

8.3.C. $\pi_{k}(Y)$ is a functional of $\Phi_{B}$ if and only if $Y \in H^{k}(B)$.

It follows from 8.3.C that $\pi_{k}(Y)$ is splittable if and only if $Y$ is tight.

\section{APPENDIX}

Square-integrable functionals of a Gaussian random field.

0.1 . Let $(\Omega, \mathscr{F}, P)$ be a probability space. We consider symmetric realvalued functions of $k$ variables $\omega^{1}, \ldots, \omega^{k} \in \Omega$ and we put

$$
(X, Y)=k ! \int_{\Omega} \ldots \int_{\Omega} X\left(\omega^{1}, \ldots, \omega^{k}\right) Y\left(\omega^{1}, \ldots, \omega^{k}\right) P\left(d \omega^{1}\right) \ldots P\left(d \omega^{k}\right)
$$

Functions $X$ for which $(X, X)<\infty$ form a Hilbert space $\Gamma^{k}$. The space $\Gamma^{0}$ consists of constants and $\Gamma^{1}$ is identical with $L^{2}(\Omega, \mathscr{F}, P)$. Let $\Gamma$ stand for the set of all sequences $X=\left(X_{0}, \ldots, X_{k}, \ldots\right), X_{k} \in \Gamma^{k}$ such that

$$
\sum_{0}^{\infty}\left(X_{k}, X_{k}\right)<\infty \text {. }
$$

Then $\Gamma$ is a Hilbert space with respect to the scalar product

$$
(X, Y)=\sum_{0}^{\infty}\left(X_{k}, Y_{k}\right)
$$

Let $X \in \Gamma^{k}, Y \in \Gamma^{l}$. Put

$$
\begin{aligned}
X \vee Y\left(\omega^{1}, \ldots, \omega^{k+l}\right) & \\
& =\frac{1}{(k+l) !} \sum X\left(\omega^{s(1)}, \ldots, \omega^{s(k)}\right) Y\left(\omega^{s(k+1)}, \ldots, \omega^{s(k+l)}\right)
\end{aligned}
$$

where the sum is taken over all permutations $s$ of indexes $(1,2, \ldots, k+l)$. It is easy to check that $X \vee Y$ belongs to $\Gamma^{k+l}$. For two arbitrary elements of 
$\Gamma$, we set

$$
X \vee Y=\sum_{n=0}^{\infty} \sum_{k=0}^{n} X_{k} \vee Y_{n-k}
$$

With respect to the operation (0.5), $\Gamma$ is an associative commutative algebra. We call it the Fock algebra over $(\Omega, \mathscr{F}, P)$.

The exponential mapping of $\Gamma^{1}=L^{2}(\Omega, \mathscr{F}, P)$ into $\Gamma$ is defined by the formula

$$
\exp Y=\sum_{k=0}^{\infty} \frac{1}{k !} Y^{[k]}
$$

where $Y^{[k]}$ is the $k$ th power of $Y$ in the algebra $\Gamma$. It follows from (0.4) that

$$
Y^{[k]}\left(\omega^{1}, \ldots, \omega^{k}\right)=Y\left(\omega^{1}\right) \ldots Y\left(\omega^{k}\right)
$$

and by (0.1) and (0.3)

$$
(\exp X, \exp Y)=e^{(X, Y)} .
$$

0.2. Let $\Phi=\left\{\varphi_{u}, u \in U\right\}$ be a random field on $(\Omega, \mathscr{F}, P)$. We denote by $\Gamma_{\Phi}$ the minimal closed subalgebra of the Fock algebra $\Gamma$ which contains all elements $\varphi_{u}, u \in U$ and we put $\Gamma_{\Phi}^{k}=\Gamma_{\Phi} \cap \Gamma^{k}$. Obviously $\Gamma_{\Phi}^{l}$ coincides with the subspace $H_{\Phi}$ of $L^{2}(\Omega, \mathscr{F}, P)$ linearly generated by $\varphi_{u}, u \in U$.

THEOREM 0.1." If $\Phi$ is a Gaussian random field, then there exists a one-to-one mapping $\pi$ of $\Gamma_{\Phi}$ onto the space $L_{\Phi}$ of all square-integrable functionals of $\Phi$ such that

0.2.A. $\pi(X)=X$ for $X \in H_{\Phi}$.

0.2.B. $E \pi\left(Y_{1}\right) \pi\left(Y_{2}\right)=\left(Y_{1}, Y_{2}\right)$ for all $Y_{1}, Y_{2}$, of $\Gamma_{\Phi}$.

0.2.C. $\pi(\exp X)=e^{X-\frac{1}{2}(X, X)}$ for $X \in H_{\Phi}$.

0.2.D. $\pi(Y)$ is a functional of a subfield $\Psi$ of the field $\Phi$ if and only if $Y$ belongs to $\Gamma_{\Psi}$.

Proof consists of the following steps: (i) We define $\pi$ on the set $Q=$ $\exp H_{\Phi}$ by the formula 0.2.C and we check that 0.2.B holds for all $Y_{1}, Y_{2}$ of $Q$. Hence $\pi$ can be continued in a unique way to an isometry of the Hilbert space $\hat{Q}$ linearly generated by $Q$ onto the Hilbert space $\hat{L}$ linearly generated by $\pi(Q)$. (ii) We prove that $\hat{Q}=\Gamma_{\Phi}, \hat{L}=L_{\Phi}$. (iii) We check the properties 0.2.A and 0.2.D.

Step 1. Every $X$ of $H_{\Phi}$ is a normal random variable with mean 0 . Hence

$$
E e^{X}=e^{\frac{1}{2} E x^{2}}=e^{\frac{1}{2}(X, X)}
$$

If $Y_{1}=\exp X_{1}, Y_{2}=\exp X_{2}, X_{1}, X_{2} \in H_{\Phi}$, then, by 0.2.C, $\pi\left(Y_{1}\right) \pi\left(Y_{2}\right)=$ $e^{X_{1}+X_{2}} e^{-\frac{1}{2}\left(X_{1}, X_{1}\right)-\frac{1}{2}\left(X_{2}, X_{2}\right)}$ and by (0.9)

$$
E \pi\left(Y_{1}\right) \pi\left(Y_{2}\right)=e^{\frac{1}{2}\left(X_{1}+X_{2}, X_{1}+X_{2}\right)} e^{-\frac{1}{2}\left(X_{1}, X_{1}\right)-\frac{1}{2}\left(X_{2}, X_{2}\right)}=e^{\left(X_{1}, X_{2}\right)}
$$

Formula 0.2.B follows from (0.10) and (0.8).

\footnotetext{
"Different forms of this theorem have been proved by Kakutani [16], Ito [15] and Segal [20]. The idea to use the exponential mapping is due to Neveu [24].
} 
Step 2. Suppose that $Y \in \Gamma_{\Phi}$ is orthogonal to $\hat{Q}$. Then for all $X \in H_{\Phi}$

$$
0=(Y, \exp X)=\sum_{0}^{\infty} 1 / k !\left(Y_{k}, X^{[k]}\right) \text {. }
$$

This implies that $Y_{k}$ is orthogonal to all products $\varphi_{u_{1}} \vee \cdots \vee \varphi_{u_{k}}$. Hence $Y=0$ and $\Gamma_{\Phi}=\hat{Q}$.

Now let $F \in L_{\Phi}$ be orthogonal to $\hat{L}$. For every $X \in H_{\Phi}$, the function $h(t)=E F e^{t X}$ is analytic in $t$. If it vanishes for all real $t$, it vanishes for all complex $t$ as well. In particular,

$$
E F e^{i X}=0 \text { for all } X \in H_{\Phi} .
$$

Put $Y \in \mathcal{W}$ if $Y$ is bounded and if $E F Y=0$. By (0.11), $Q$ contains the family $e^{i X}, X \in H_{\Phi}$ which is closed under multiplication. Besides $\mathscr{W}$ is a linear space; it contains with each function the complex conjugate of this function and with each uniformly bounded convergent sequence the limit of this sequence. This implies (see [19, Chapter 1, Theorem 2] or [5, Lemma 1.2]) that 2 contains all bounded functions measurable with respect to the $\sigma$-algebra generated by $e^{i X}$. Hence $F$ is orthogonal to all bounded functionals of the field $\Phi$, and $F=0$ a.s. $P$. This proves the equality $L=\hat{L}$.

Step 3. Fix $x \in H_{\Phi}$ and put $F_{t}=\exp t X$. It follows from (0.6) that $\lim _{t \rightarrow 0} t^{-1}\left(F_{t}-1\right)=X$ in $\Gamma$. Hence $t^{-1}\left(\pi\left(F_{t}\right)-1\right) \rightarrow \pi(X)$ in $L_{\Phi}$. By 0.2.C $\pi\left(F_{t}\right)=e^{i X-\frac{1}{2} t^{2}(X, X)}$. Hence $\pi(X)=X$.

The property 0.2.D follows from the fact that $\Gamma_{\Psi}$ is linearly generated by $\exp H_{\Psi}$ and $L_{\Psi}$ is linearly generated by $\pi\left(\exp H_{\Psi}\right)$ which is true since the reasoning of Step 2 is applicable to $\Psi$ as well as to $\Phi$.

0.3. To prove Theorem 8.1, it is sufficient to define, for every $k$, a mapping $\gamma_{k}$ of $H^{k}$ onto $\Gamma_{\Phi}^{k}$ in such a way that

0.3.A. $\left(\gamma_{k} Y_{1}, \gamma_{k} Y_{2}\right)=E Y_{1} Y_{2}$ for every $Y_{1}, Y_{2} \in H^{k}$.

0.3.B. For every set $B, \gamma_{k} H^{k}(B)=\Gamma_{\Phi_{B}}^{k}$.

It follows from 0.2.A, B, C, D and 0.3.A and B that the mappings $\pi_{k}=\pi \gamma_{k}$ satisfy conditions 8.1.A, B, C.

0.4. For every $\mu_{1}, \ldots, \mu_{k} \in \Re(E)$, we put

$$
\mu_{1} \vee \cdots \vee \mu_{k}=1 / k ! \sum_{s \in S} \mu_{s}(1) \times \cdots \times \mu_{s}(k)
$$

Let $Q$ be the set of all $\varphi_{\mu}$ corresponding to measures $\mu$ of the form (0.12). Put

$$
\gamma_{k} \varphi_{\mu_{1} \vee \cdots \vee \mu_{k}}=\frac{1}{\sqrt{k !}} \varphi_{\mu_{1}} \vee \cdots \vee \varphi_{\mu_{k}}
$$

where $\bigvee$ is the operation defined by $(0.4)$ and $(0.5)$. By $(0.1)$,

$$
\left(\varphi_{\mu_{1}} \vee \cdots \vee \varphi_{\mu_{k}}, \varphi_{r_{1}} \vee \cdots \vee \varphi_{r_{k}}\right)=\sum_{s} E \varphi_{\mu_{1}} \varphi_{r_{k}(1)} \ldots E \varphi_{\mu_{k}} \varphi_{r_{\gamma_{k}(k)}} \text {. }
$$

On the other hand, by (0.12) and (1.5),

$$
\begin{aligned}
E \varphi_{\mu_{1} \vee \cdots \vee \mu_{k}} \varphi_{\nu_{1} \vee \cdots \vee v_{k}} & =\left\langle\mu_{1} \vee \cdots \vee \mu_{k}, \nu_{1} \vee \cdots \vee \nu_{k}\right\rangle \\
& =1 / k ! \sum_{s}\left\langle\mu_{1}, \nu_{s(1)}\right\rangle \cdots\left\langle\mu_{k}, \nu_{s(k)}\right\rangle .
\end{aligned}
$$

It follows from (0.13), (0.14) and (0.15) that 0.3.A holds for all $Y_{1}, Y_{2}$ of $Q$. 
We continue the mapping $\gamma_{k}$ to an isomorphic mapping of the space $\hat{Q}$ linearly generated by $Q$ onto the space $\Gamma_{\Phi}^{k}$ which is linearly generated by elements (0.13). It remains to show that $\hat{Q}=H^{k}$ and then to verify 0.3.B.

0.5. LEMMA 0.1. Let $\hat{H}$ stand for the subspace of $H\left(E^{k}\right)$ linearly generated by

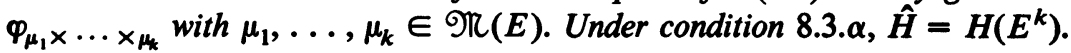

Proof. Put $\mu \in \hat{\mathfrak{N}}$ if $\varphi_{\mu} \in \hat{H}$. According to subsection 3.3 , the equality $\hat{\mathfrak{N}}=\Re$ will be proved if we show that, for every finite measure $\mu \in \mathfrak{N}$, the set $\Re$ contains the measures $\mu_{\delta}$ defined by (3.3). We note that

$$
\mu_{\delta}(B)=\int_{E^{k}} \mu(d x) m_{\delta x}(B)
$$

where

$$
m_{\delta x}(B)=\int_{B} p_{\delta}(x, y) m(d y) .
$$

By (1.4), 3.1.B and 8.3. $\alpha$,

$$
\left\langle m_{\delta x}, m_{\delta x}\right\rangle=g(\delta, x, x)<\infty .
$$

Hence $m_{\delta x} \in \mathfrak{N}$. Obviously $m_{\delta x} \in \hat{\mathfrak{T}}$.

For every $Y \in H\left(E^{k}\right)$, the expectation $E Y \varphi_{m_{a x}}$ is measurable in $x$. Hence (see e.g. [14, §3.2]), for every $\varepsilon>0$, there exists a partition of $E^{k}$ into disjoint sets $C_{1}, \ldots, C_{k}, \ldots$, such that

$$
E\left(\varphi_{m_{0 x}}-\varphi_{m_{0 y}}\right)^{2}<\varepsilon^{2} \text { if } x, y \in C_{n}, n=1,2, \ldots
$$

Choose an arbitrary point $c_{n}$ of $C_{n}$ and put $\hat{m}_{\delta x}=m_{\delta c_{n}}$ for $x \in C_{n}$. By (0.18) and (1.5)

$$
\left\|m_{\delta x}-\hat{m}_{\delta x}\right\|<\varepsilon \text { for all } x \in E^{k} .
$$

By subsection 3.3, the restriction of $\mu$ to $E_{n}=C_{1} \cup \cdots \cup C_{n}$ converges to $\mu$ in $M$. Therefore, without loss of generality, we can assume that $\mu$ is concentrated on $E_{n}$. Put

$$
\hat{\mu}_{\delta}(B)=\int_{E^{k}} \mu(d x) \hat{m}_{\delta x}(B)=\sum_{k=1}^{n} \mu\left(C_{k}\right) m_{\delta c_{k}}(B) .
$$

By (1.4), (0.16) and (0.19),

$$
\begin{aligned}
\left\langle\mu_{\delta}-\hat{\mu}_{\delta}, \mu_{\delta}-\hat{\mu}_{\delta}\right\rangle & =\int_{E^{k} \times E^{k}} \mu(d x)\left\langle m_{\delta x}-\hat{m}_{\delta x}, m_{\delta x}-\hat{m}_{\delta x}\right\rangle \mu(d y) \\
& <\mu\left(E^{k}\right)^{2} \varepsilon^{2}
\end{aligned}
$$

By $(0.20), \hat{\mu}_{\delta} \in \hat{M}$. Hence $\mu_{\delta} \in \hat{M}$.

0.6. It follows from Lemma 0.1 that the elements $\varphi_{\mu_{1} \vee} \cdots \vee \mu_{k}, \mu_{1}, \ldots, \mu_{k} \in$ $\mathfrak{T}(E)$ linearly generate $H^{k}$. Hence their orthogonal projections on $H^{k}(B)$ linearly generate $H^{k}(B)$. It follows from subsections 7.2, 7.3, that the orthogonal projection of $\varphi_{\mu_{1} \vee \cdots \vee \mu_{k}}$ on $H^{k}(B)$ has a form $\varphi_{\tilde{\mu}_{1} \vee \cdots \vee \tilde{\mu}_{k}}, \tilde{\mu}_{1}, \ldots, \tilde{\mu}_{k} \in$ IR $(B)$. Now the property 0.3.B follows from (0.13).

\section{REFERENCES}

1. P. Billingsley, Probability and measure, Wiley, New York, 1979.

2. R. M. Blumenthal and R. K. Getoor, Markov processes and potential theory, Academic Press, New York, 1968. 
3. R. Cairoli and J. B. Walsh, Stochastic integrals in the plane, Acta Math. 134 (1975), 111-183.

4. R. L. Dobrushin and M. Ja. Kelbert, Local additive functionals of Gaussian generalized fields, Uspehi Mat. Nauk 34 (1979), No. 5, 223-224. (Russian)

5. E. B. Dynkin, Theory of Markov processes, Pergamon Press, Oxford, 1960.

6. __ Markov processes, Vols. I and II, Springer-Verlag, Berlin-Göttingen-Heidelberg, 1965.

7. __ Markov processes. Theorems and problems, Plenum, New York, 1969.

8. __ Regular Markov processes, Uspehi Mat. Nauk 28 (1973), No. 2, 35-64. (English translation: Russian Math. Surveys 28, (1973), No. 2, 33-64)

9. __ Markov systems and their additive functionals, Ann. Probability 5 (1977), 653-677.

10. __ Additive functionals of non-interacting Markov processes (in preparation).

11. M. Fukushima, Dirichlet forms and Markov processes, North-Holland, Amsterdam, Oxford, New York, 1980. Kodashira, Tokyo.

12. R. K. Getoor, Markov processes: Ray processes and right processes, Lecture Notes in Math., vol. 440, Springer-Verlag, Berlin and New York, 1975.

13. M. Hall, Jr., Combinatorial theory, Blaisdell, Waltham, Mass., 1967.

14. E. Hille, Functional analysis and semigroups, New York, 1948.

15. K. Ito, Multiple Wiener integral, J. Math. Soc. Japan 3 (1951), 157-169.

16. S. Kakutani, Determination of the spectrum of the flow of Brownian motion, Proc. Nat. Acad. Sci. U.S.A. 36 (1950), 319-323.

17. S. Karlin and H. M. Taylor, A first course in stochastic processes, 2nd ed., Academic Press, New York, 1975.

18. H. McKean, Brownian motion with a several-dimensional time, Teor. Verojatnost. i Primenen. 8 (1963), 357-378 = Theor. Probability Appl. 3 (1963), 335-354.

19. P. A. Meyer, Probability and potentials, Blaisdell, Waltham, Mass., 1966.

20. G. M. Molchan, Characterization of Gaussian fields with Markovian property, Dokl. Acad. Nauk SSSR 197 (1971), No. 4, 784-787. (English translation: Soviet Math. Dokl. 12 (1971), No. 2, 563-567)

21. _ L-Markov Gaussian fields, Dokl. Acad. Nauk SSSR 215 (1974), No. 5, 1054-1057. (English translation: Soviet Math. Dokl. 15 (1974), No. 2, 657-662)

22. E. Nelson, Construction of quantum fields from Markov fields, J. Functional Analysis 12 (1973), 97-112.

23. ___ The free Markov field, J. Functional Analysis 12 (1973), 211-227.

24. J. Neveu, Processus aléatoires Gaussiens, Les Presses de l'Université de Montréal, Montréal, 1968.

25. S. Orey and W. Pruitt, Sample functions of the N-parameter Wiener process, Ann. Probability 1 (1973), 138-163.

26. I. Segal, Tensor algebras over Hilbert spaces. I, Trans. Amer. Math. Soc. 81 (1956), 106-134.

27. M. L. Silverstein, Symmetric Markov processes, Lecture Notes in Math., vol. 426, SpringerVerlag, Berlin, Heidelberg, New York, 1974.

28. B. Simon, The $\boldsymbol{P}(\Phi)_{2}$ Euclidean (quantum) field theory, Princeton Univ. Press, Princeton, N. J., 1974.

29. J. B. Walsh, Martingales with a multi-dimensional parameter and stochastic integrals in the plane, Lecture Notes, Université P.\&M. Curie, Paris, 1976/1977.

30. R. L. Wolpert, Wiener path intersections and local time, J. Functional Analysis 30 (1978), 329-340.

31. __ Local time and a particle picture for Euclidean field theory, J. Functional Analysis 30 (1978), 341-357.

32.

33. E. Wong and M. Zakai, Martingales and stochastic integrals for processes with a multi-dimensional parameter, Z. Wahrscheinlichkeitstheorie und Verw. Gebiete 29 (1974), 109-122.

34. J. Yeh, Wiener measure in a space of functions of two variables, Trans. Amer. Math. Soc. 95 (1960), 433-450.

Department of Mathematics, Cornell University, Ithaca, New York 14853 
